Article

\title{
Exploring the Full Potential of Reversible Deactivation Radical Polymerization Using Pareto-Optimal Fronts
}

\author{
Stijn K. Fierens ${ }^{1}$, Dagmar R. D'hooge ${ }^{1,2}$, Paul H. M. Van Steenberge ${ }^{1}$, \\ Marie-Françoise Reynierss ${ }^{1, *}$ and Guy B. Marin ${ }^{1}$
}

1 Laboratory for Chemical Technology (LCT), Ghent University, Technologiepark 914, B-9052 Zwijnaarde (Gent), Belgium; E-Mails: stijn.fierens@ugent.be (S.K.F.); Dagmar.Dhooge@ugent.be (D.R.D.); paul.vansteenberge@ugent.be (P.H.M.V.S.); guy.marin@ugent.be (G.B.M.)

2 Department of Textiles, Ghent University, Technologiepark 907, B-9052 Zwijnaarde (Gent), Belgium

* Author to whom correspondence should be addressed;

E-Mail: mariefrancoise.reyniers@ugent.be; Tel.: +32-9-331-1754; Fax: +32-9-331-1759.

Academic Editor: Philipp Vana

Received: 27 February 2015 / Accepted: 1 April 2015 / Published: 9 April 2015

\begin{abstract}
The use of Pareto-optimal fronts to evaluate the full potential of reversible deactivation radical polymerization (RDRP) using multi-objective optimization (MOO) is illustrated for the first time. Pareto-optimal fronts are identified for activator regenerated electron transfer atom transfer radical polymerization (ARGET ATRP) of butyl methacrylate and nitroxide mediated polymerization (NMP) of styrene. All kinetic and diffusion parameters are literature based and a variety of optimization paths, such as temperature and fed-batch addition programs, are considered. It is shown that improvements in the control over the RDRP characteristics are possible beyond the capabilities of batch or isothermal RDRP conditions. Via these MOO-predicted non-classical polymerization procedures, a significant increase of the degree of microstructural control can be obtained with a limited penalty on the polymerization time; specifically, if a simultaneous variation of various polymerization conditions is considered. The improvements are explained based on the relative importance of the key reaction rates as a function of conversion.
\end{abstract}


Keywords: controlled radical polymerization; model-based design; multi-objective optimization; Pareto; kinetic modeling; ARGET ATRP; NMP

\section{Introduction}

During the last two decades, reversible deactivation radical polymerization (RDRP), which is also known as controlled radical polymerization (CRP), has shown to overcome disadvantages of conventional free radical polymerization (FRP), which allows mostly the synthesis of commodity polymer products [1-7], unless expensive functional monomers are used [8,9]. Under well-defined conditions, RDRP techniques are characterized by the establishment of a dynamic pseudo-equilibrium between propagating and dormant species, allowing the controlled incorporation of monomer units per activation-growth-deactivation cycle. This enables the production of polymers with a predetermined number average chain length and narrow chain length distribution (CLD; dispersity $(\bigoplus)<1.3)$ that possess end-group functionality (EGF). This brings the synthesis of well-defined macromolecular architectures, such as block and star copolymers, within reach. Two important RDRP techniques, which are studied in this work, are activators regenerated by electron transfer atom transfer radical polymerization (ARGET ATRP) [10-16] and nitroxide mediated polymerization (NMP) [17-21].

In traditional ATRP (Figure 1; left top), typically a $\mathrm{Cu}(\mathrm{I})$-complex $\left(C u^{I} X / L\right.$; activator; $\mathrm{X}$ : halogen atom) catalyzes the homolytic cleavage of an ATRP initiator $\left(R_{0} X^{\prime}\right)$ to yield an initiator radical $\left(R_{0}^{\prime}\right)$ and a transition metal complex characterized by a higher oxidation state $\left(C u^{I I} X_{2} / L\right.$; deactivator). This $R_{0}$ species propagates until it is temporarily deactivated by $C u^{I I} X_{2} / L$ to yield a halide capped dormant macrospecies $\left(R_{i} X ; i\right.$ : chain length). Typical monomers are styrene, (meth)acrylates and acrylamides. However, during the initial stage of the ATRP, termination reactions are also occurring at significant rates, thereby leading to a build-up of deactivator species. Hence, the deactivation reaction is favored over the termination reaction at higher polymerization times, allowing microstructural control. This effect has been first described by Fischer et al. [22,23] and is known as the persistent radical effect [24]. The commercial utilization of ATRP is, however, hampered by various challenges such as perfect oxygen removal, excessively high catalyst concentrations leading to extensive post-polymerization purifications, and the toxic characteristics of the transition metal complexes [1]. Therefore, alternative initiating procedures which result in a much more attractive ATRP have been investigated, i.e., systems in which the catalyst concentration is dramatically lowered and less stringent reaction conditions are employed [25].

One of these modified ATRPs is ARGET ATRP (Figure 1; left panel) [26-28]. In this RDRP, a reducing agent, for example tin(II) 2-ethylhexanoate $\left(\operatorname{Sn}(E H)_{2}\right)$, is added in excess relative to the ATRP catalyst, which is present at very low amounts ( $<300 \mathrm{ppm}$ with respect to monomer; molar). This allows the in situ reduction of the deactivator species and thus the regeneration of activator species. Moreover, ARGET ATRP allows the polymerization to be initiated by only the halide initiator and the deactivator, leading to less stringent reaction conditions compared to the classical ATRP process. It has been indicated that the outcome of the ARGET ATRP is very sensitive to the initial concentrations. In particular, the amount of reducing agent has to be selected carefully, taking into account a trade-off 
between polymerization rate and livingness [29-32]. Moreover, by switching to continuous operation, a more industrial attractive process can be obtained [33,34].

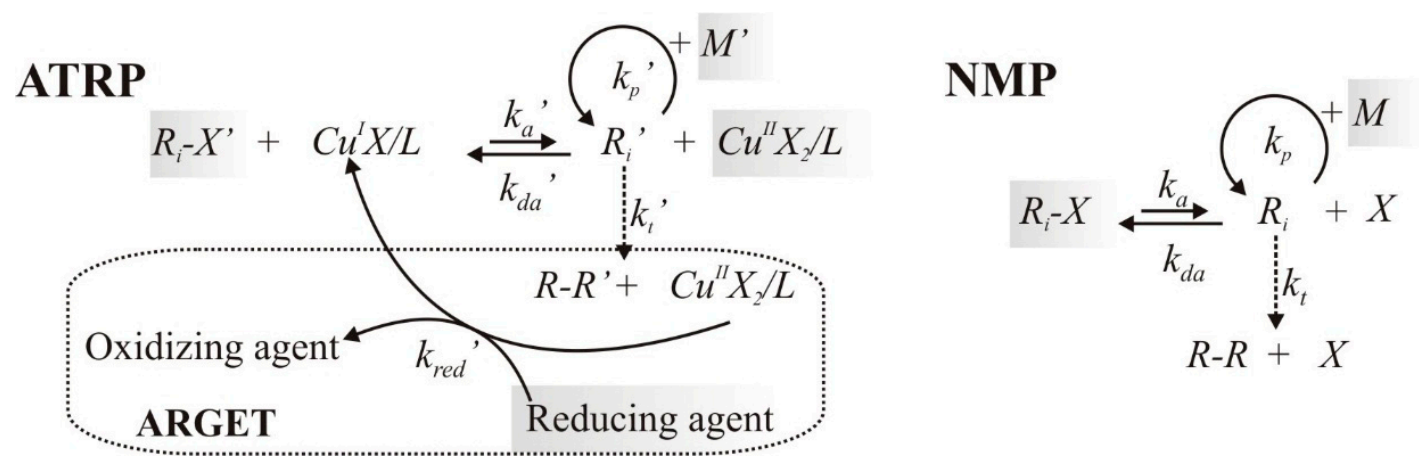

Figure 1. Left: basic reaction scheme for Activators ReGenerated by Electron Transfer (ARGET) Atom Transfer Radical Polymerization (ATRP) using a Cu-based catalyst ( $C u^{I I} X_{2} / L$; deactivator) and as ATRP initiator $R_{0} X^{\prime}$ (X: halogen atom), and monomer $M$; Right: basic reaction scheme for Nitroxide Mediated Polymerization (NMP) using an alkoxyamine initiator $R_{0} X$ and monomer $M$; $k$ : rate coefficient; $a, d a, p, r e d, t$ : activation, deactivation, propagation, reduction, and termination; $i$ : chain length with $i=0$ initiator related; grey box: initial presence of this species $(i=0)$; for simplicity only termination by recombination is shown; in traditional ATRP no reducing agent and also activator present at the start.

The basic kinetic scheme of the second studied RDRP, i.e., NMP [17,35], is shown in Figure 1 (right panel). NMP is one of the first discovered RDRP systems, due to the pioneering work of Rizzardo et al. [36]. Since then, numerous studies have been devoted to clarify the mechanism and the kinetics of NMP [37-42], focusing mostly on styrene and acrylates as monomers. In NMP, an alkoxyamine initiator $\left(R_{0} X\right)$, undergoes a homolytic cleavage at elevated temperature, which releases a $R_{0}$ species and a nitroxide radical $(X)$, also called the persistent radical. As in ATRP, the initiator radical undergoes chain initiation and the persistent radical effect takes place allowing control at higher polymerization times [43]. Further optimization can be obtained via, for example, novel and highly active nitroxides/alkoxyamines [44,45], such as $N$-(2-methyl-2-propyl)- $N$-(1-diethylphosphono-2,2-dimethylpropyl)- $N$-oxyl (MAMA-SG1; BlocBuilder $\left.{ }^{\circledR}\right)$, the use of conventional radical initiators with a long half-life time [46], the continuous addition of conventional radical initiator [47], and rate-enhancing organic acids [48].

However, both ARGET ATRP and NMP still display several shortcomings, which partly explains the limited industrial realization of RDRP [49]. Taking into account technical-economic considerations, several aspects should be still optimized. From a technical point of view an improved polymer product, i.e., a better control over the narrowness of the CLD and a high EGF, are desired. On the other hand, the economic feasibility of the polymerization process necessitates that a predetermined conversion is reached within a minimal reaction time. These objectives are unfortunately often contradictory in nature, implying the need of multi-objective optimization (MOO) for the unbiased optimization of RDRP. Such optimization studies do not deliver a unique set of operating conditions, but result in a set of several equivalently optimal solutions. This so-called Pareto-optimal front offers the decision-maker useful insights in the compromise to be made and provides the flexibility to decide which is the preferred 
operating point taking into account specific constraints on polymerization time and microstructural control [50,51].

Several approaches have been developed that are suited for solving MOO-problems, such as fuzzy logic, neural networks, simulated annealing, and genetic algorithms [52]. The latter have shown to be very suited to solve MOO-problems involving many design variables, as in RDRP. A pioneering genetic algorithm is the so-called Nondominated Sorting Genetic Algorithm (NSGA) proposed by Srinivas and Deb [53]. This algorithm suffered from several drawbacks, which have been addressed by the development of an improved version NSGA-II [54].

MOO has already been successfully applied for several conventional radical and non-radical polymerization processes, such as the production of nylon-6 in a semi-batch operated reactor [55], the synthesis of polyester films [56], semi-batch epoxy polymerization [57], free radical (co)polymerization [50,58], and emulsion polymerization [59,60]. Typically off-line optimization is performed, due to limitations for the calculation time of the MOO algorithm for complex kinetic schemes. For RDRP, however, this methodology has not yet been explored. In this work, for the first time, the potential of MOO for RDRP will be illustrated, considering ARGET ATRP of butyl methacrylate (BMA) and NMP of styrene as model cases and using NSGA-II [61-63]. All kinetic and diffusion parameters are taken from literature [61,64,65] and a variety of optimization paths, such as temperature and fed-batch addition programs, are included, considering time, $Ð$ and EGF as objectives. For simplicity, the objectives are evaluated only at a final conversion of 0.75 . It is shown that significant progress can be made in the control over the RDRP characteristics via such non-classical polymerization procedures.

The reported improvements are explained by a comparison of the relative importance of the key reaction rates as a function of conversion. This involves a comparison of the propagation, deactivation and termination reaction probabilities and in addition probabilities related to important side reactions, such as chain transfer to dimer in the case of NMP of styrene.

\section{Modeling Procedure}

\subsection{Reaction Schemes and Rate Coefficients}

The reaction scheme for ARGET ATRP of BMA using $S n(E H)_{2}$ as reducing agent ( $R^{I I}$ in Table 1), ethyl 2-bromoisobutyrate as ATRP initiator $\left(R_{0} X^{\prime}\right.$ in Table 1), and $C_{u B r} / T P M A$ (TPMA: tris[(2-pyridyl)methyl]amine; $D^{\prime}$ in Table 1) as deactivator is given in Table 1 (left column), considering bulk conditions for simplicity. The listed kinetic parameters are adopted from Payne et al. [64] and a distinction is made between ARGET ATRP specific and non-specific reaction steps. The main reactions are propagation, termination, and ATRP (de)activation. Both termination by disproportionation and recombination are considered. Chain transfer to monomer is neglected, based on previous studies [66]. Furthermore, a first and second reduction step for activator regeneration is included. Chain length and conversion dependent apparent termination rate coefficients are calculated via the composite $k_{t}$-model using the Reversible Addition Fragmentation Transfer-Chain Length Dependent-Termination method (RAFT-CLD-T), with improved parameters taken from the recent work from Derboven et al. [65]. For the other reaction steps, no diffusional limitations have to be accounted for, at least to a first 
approximation, as typically a maximal conversion of 0.75 is obtained. Note that the MOO-strategy can be extended to higher conversions, provided that reliable activation/deactivation parameters become available. A deterministic model based on the method of moments is used to describe the polymerization kinetics. More details on the method of moments can be found in Supporting Information.

Table 1. Reactions and Arrhenius parameters for the simulation of ARGET ATRP of butyl methacrylate (BMA; left column) and NMP of styrene initiated by MAMA-SG1 (middle column); Arrhenius parameters $\left(A\left(\left(\mathrm{~L} \cdot \mathrm{mol}^{-1}\right) \cdot \mathrm{s}^{-1}\right)\right.$ and $\left.E_{a}\left(\mathrm{~kJ} \cdot \mathrm{mol}^{-1}\right)\right)$ given in right column. Termination reactions are included in the kinetic model but not explicitly shown (for ARGET ATRP case: recombination and disproportionation; for NMP case: recombination); $A^{\prime}=\mathrm{Cu}^{\mathrm{I} X} / \mathrm{L}, D^{\prime}=\mathrm{Cu}^{\mathrm{II}} \mathrm{X}_{2} / \mathrm{L}, R^{z}=\mathrm{Sn}^{\mathrm{Z}}(\mathrm{EH})_{2}, M^{\prime}=$ butyl methacrylate, $R_{0} X^{\prime}=$ ethyl 2bromoisobutyrate, $M=$ styrene, $R_{0} X=$ MAMA-SG1, $D=\operatorname{dimer} ; j=1,2,3,4 ; j^{\prime}=2,3,4$.

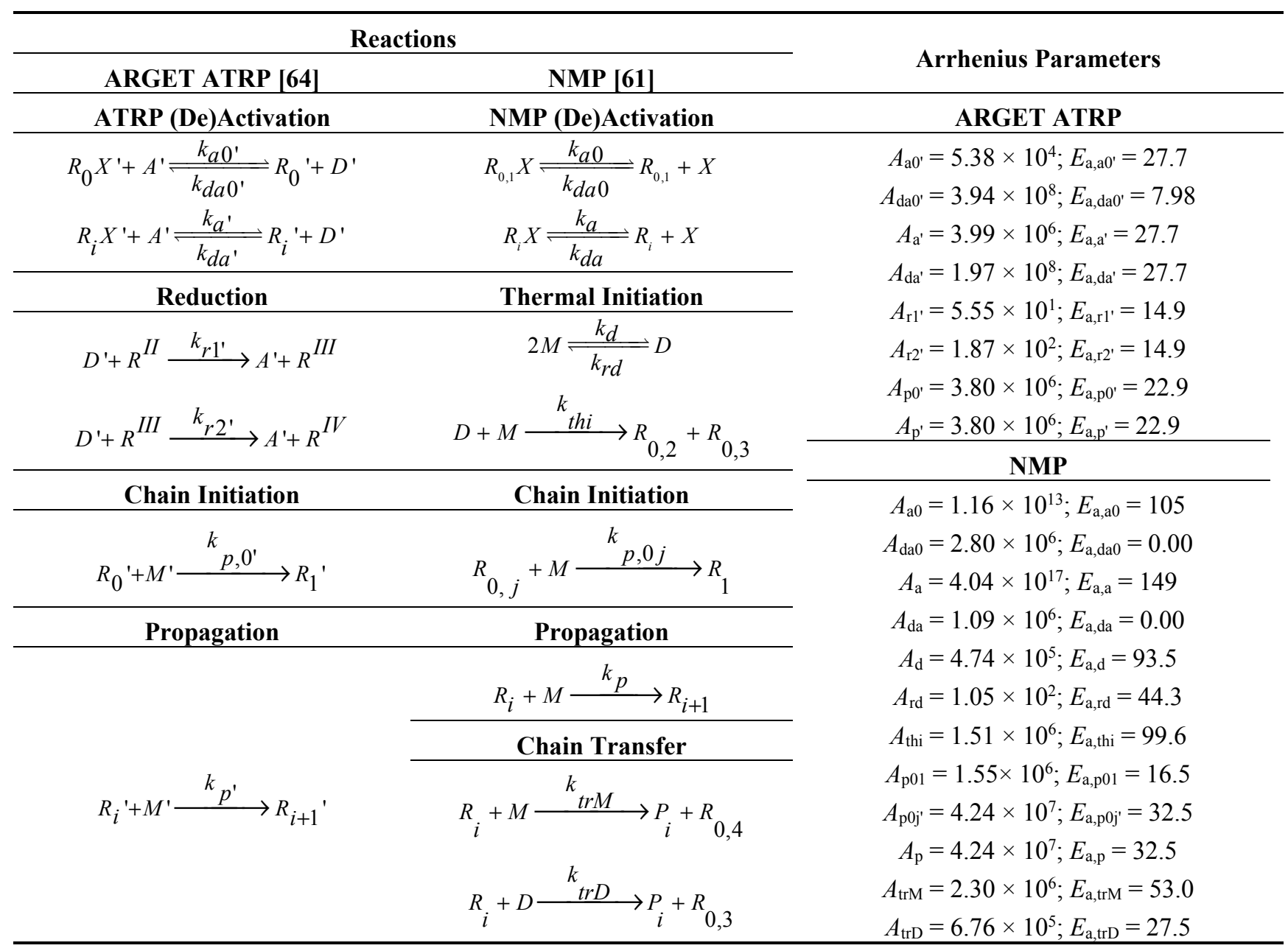

An overview of the reactions included in the kinetic model of the NMP of styrene initiated by MAMA-SG1 $\left(R_{0,1} X\right.$ in Table 1$)$ is given in Table 1 (middle column). A distinction is made between styrene and NMP specific reaction steps. The corresponding Arrhenius parameters are taken from Fierens et al. [61], who performed regression analysis on an extensive set of experimental polymerization data to obtain NMP (de)activation kinetic parameters while accounting for the reactivity difference between initiator and macrospecies. Importantly, for the styrene specific steps, thermal auto-initiation [67] is included, since for styrene, at elevated temperatures $\left(>100{ }^{\circ} \mathrm{C}\right)$, auto-initiation 
occurs in which two styrene molecules undergo a Diels-Alder cycloaddition to form a dimer molecule ( $D$ in Table 1). This dimer can undergo a retro Diels-Alder reaction forming two styrene molecules or it can undergo a molecule assisted homolysis to yield two additional initiator radicals $R_{0,2}$ and $R_{0,3}$. Chain transfer reactions to monomer and dimer are considered, based on literature data $[62,68]$. Again, chain length and conversion dependent apparent rate coefficients are taken into account $[69,70]$. Here, also, a deterministic model based on the method of moments has been used.

\subsection{Genetic Optimization Algorithm: NSGA-II}

A solution, i.e., a set of variables (e.g., temperature and molar amounts for a given conversion interval), is said to be Pareto-optimal if there exists no other set of feasible variables that will yield an improvement in one objective (e.g., $Ð$ ) without worsening at least one other objective (e.g., time to reach a given conversion). This set of conditions is said to be non-dominated and belongs to the Pareto-optimal front [52].

In this contribution, the Nondominated Sorting Genetic Algorithm-II (NSGA-II) has been implemented in FORTRAN code and combined with literature deterministic kinetic models for ARGET ATRP of BMA and the NMP of styrene to perform MOO. In the first step of the NSGA-II algorithm (Scheme S1; Supporting Information), a population of $N_{p}$ parents $\left(P_{1}\right.$; generation 1$)$ is generated. This population refers to a set of initial variables, which are randomly distributed in between minimum and maximum boundaries (e.g., a temperature range for a specific conversion). The polymerization characteristics of the population are evaluated at a predetermined conversion, after which the population is sorted based on non-domination, leading to a ranking of the individuals. This results in a so-called fitness value per population member, also known as the rank. Subsequently, each population member is assigned a crowding-distance, which allows identifying their level of remoteness in the objective space. This ensures the diversity of the population upon regeneration, which in this first generation refers to the creation of an initial offspring population $\left(Q_{i} ; i=1\right)$ of size $N_{o}$. The latter population is obtained via selection, with rank as first decision criterion and crowding-distance as second, and subsequent crossover, and mutation.

In a next step, the ranks and crowding-distances of the combined population $\left(R_{i}\right)$, i.e., parents and offspring population, are determined. Hereafter, a new generation for the parent population $\left(P_{i+1}\right)$ of $N_{p}$ members is created by selecting the best performing individuals out of the combined population with, again, the same decision criteria, i.e., rank and crowding-distance.

Additional generations are obtained by repeating the aforementioned process of offspring generation and reduction of population members to $N_{p}$ size until a predefined maximum generation number is reached. If this number is sufficiently large the Pareto-optimal front is approached, provided that the other NSGA-II parameters (Table S1; Supporting Information) are properly chosen. For more details on the NSGA-II algorithm, the reader is referred to Deb et al. [54].

An overview of the upper and lower bounds of the variables selected in this work is given in Supporting Information (Table S2). 


\section{Results and Discussion}

\subsection{Multi-Objective Optimization of ARGET ATRP}

The MOO of ARGET ATRP of BMA is explored considering time and $Ð$ as objectives. Two objectives are selected in this case study to illustrate the strength of Pareto-optimal fronts, taking into that the simulated EGF variation is rather limited $(<5 \%)$. Moreover, it has been verified that similar number averaged chain lengths are obtained for all cases considered. Several optimization pathways are considered, namely optimization via the application of a non-isothermal temperature program, fed-batch addition of monomer and fed-batch addition of reducing agent. The fed-batch addition of deactivator is ignored, since preliminary simulations revealed no improvement compared to the batch condition. Also the combination of several optimization pathways is examined and shown to be synergetic in particular cases. A constraint on the polymerization time of maximum $50 \mathrm{~h}$ is imposed and the total molar amount of reactants is fixed with as reference case the following batch conditions $\left([M]_{0} /\left[R_{0} X\right]_{0} /\left[C u^{I I} X_{2 / L}\right]_{0} /\left[S_{n}^{I I}(E H)_{2}\right]_{0}=200 / 1 / 0.005 / 0.05 ; 90^{\circ} \mathrm{C}\right)$.

\subsubsection{Variation of Temperature}

As a first individual optimization pathway, the use of a temperature program is investigated. For simplicity, a piecewise linear temperature profile is selected, consisting of six distinct conversion intervals, each of equal size $\left(\Delta X_{m}=0.125\right)$ with a maximal conversion of 0.75 . Hence, seven variables, i.e., the temperatures at the interval boundaries, have to be optimized by the MOO algorithm per simulation. Note that in practice these conversion points should be translated into times for a practical realization of the off-line optimization strategy. For completeness, it is mentioned here that also in the remainder of the text temperature/fed-batch addition programs are always described per conversion interval of $\Delta X_{m}=0.125$. In other words, the boundary values of these intervals have to be determined per simulation. In the current situation of a temperature program, a variation between 60 and $90{ }^{\circ} \mathrm{C}$ is allowed for the seven variables, based on literature data on isothermal batch ATRP of BMA [64]. The results of the MOO are shown in Figure 2a (green dots). For the sake of comparison, the Pareto-optimal front for isothermal polymerizations is also shown (red dots).

It can be seen that the use of a temperature program is beneficial compared to the isothermal case. For example, for the synthesis of a polymer product with a $Ð$ of 1.35 , a reduction of the polymerization time with $c a .3 \mathrm{~h}$ results when using a temperature program. The values of the seven variables describing the temperature program along the Pareto-optimal front are shown in Figure 2b. By linking this figure to Figure 2a it follows that the final $Ð$ decreases if the ARGET ATRP is started at a lower temperature and the temperature is gradually increased throughout the polymerization. This increased control over chain length is although accompanied by a longer polymerization time. This becomes clearer when selecting three illustrative cases along the Pareto-optimal front (cases 1, 2 and 3 in Figure 2a,b). For completeness, the complete temperature programs for these three cases are shown in Figure 2c. The most dynamic program is obtained for case 1 , whereas case 2 corresponds to a less dynamic T-program providing a transition to the limiting case 3, in which an isothermal ARGET ATRP is conducted.

The observed differences in polymerization time and $Ð$ can be explained by looking at the ratio of the reaction probability for a macroradical to propagate and to deactivate $\left(P_{\text {prop }} / P_{\text {deac }}\right)$ (Figure $\left.2 \mathrm{~d}\right)$ on one 
hand and the reaction probability for a macroradical to terminate $\left(P_{\text {term }}\right)$ (Figure 2e) on the other hand. The former characteristic is selected since simplified models assuming perfect livingness have shown that the ratio of the propagation and deactivation reactivity need to be sufficiently low to guarantee the incorporation of a controlled amount of monomer units during each activation-propagation-deactivation cycle so that a low $D$ can be obtained [1,71]. In reality, a perfect livingness cannot be achieved since termination is inevitable and thus $P_{\text {term }}$ has to be considered as a second characteristic [72,73]. Note that no additional characteristics are needed since other side reactions can be neglected [64].

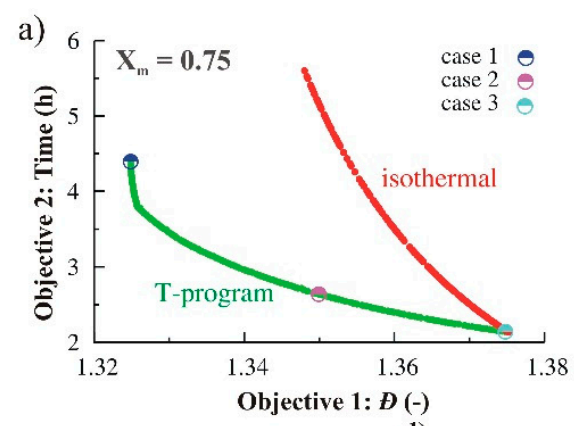

c)

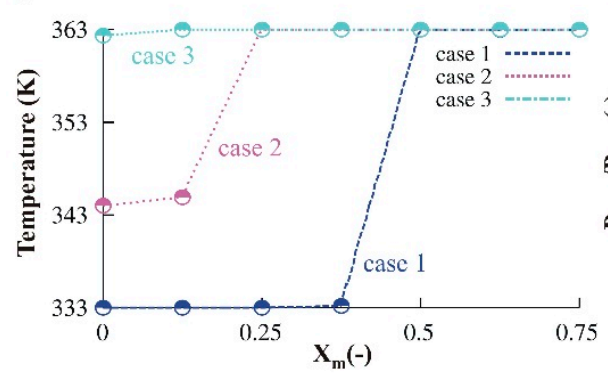

d)

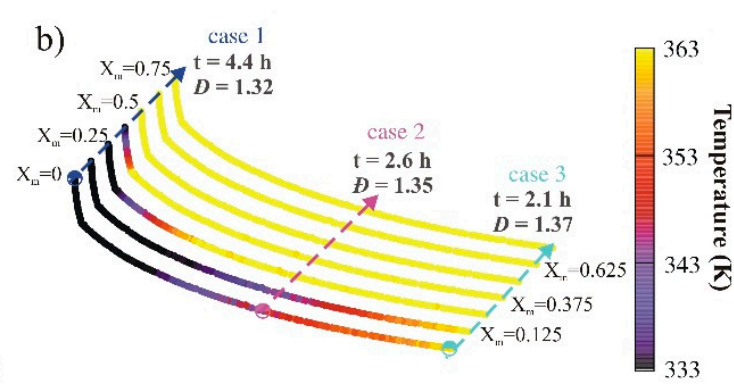

e)
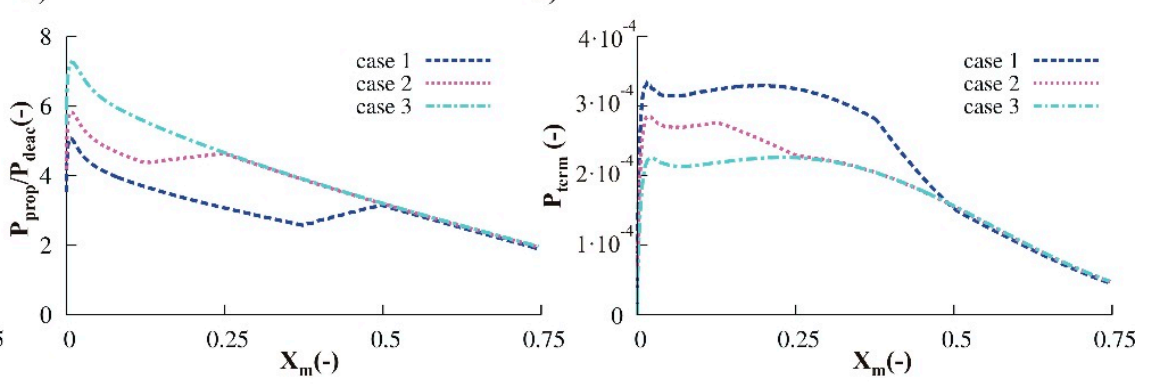

Figure 2. Results for the multi-objective optimization (MOO) of ARGET ATRP of BMA using a piecewise linear temperature program $\left(\Delta X_{m}=0.125\right.$ until $X_{m}=0.75$; $[M]_{0} /\left[R_{0} X\right]_{0} /\left[C u^{I I} X_{2} / L\right]_{0} /\left[S^{I I}(E H)_{2}\right]_{0}=200 / 1 / 0.005 / 0.05$ ) (a) Pareto-optimal front (green circles) and corresponding isothermal front (red circles); (b) Variation of the temperature at the interval boundaries along the Pareto-optimal front; (c) Temperature as a function of $X_{m}$ for three selected cases in (a); (d) Ratio of probability for propagation to deactivation as a function of $X_{m}$ for the selected cases; and (e) Probability for termination as a function of $X_{m}$ for the selected cases.

Figure $2 \mathrm{~d}$ shows that at low conversions a lower polymerization temperature clearly leads to a lower $P_{\text {prop }} / P_{\text {deac }}$ and thus a slower ARGET ATRP and a better control over chain length. This can be understood by noting that propagation is much more activated $\left(23 \mathrm{~kJ} \cdot \mathrm{mol}^{-1}\right.$ in Table 1$)$ than deactivation $\left(8 \mathrm{~kJ} \cdot \mathrm{mol}^{-1}\right.$ in Table 1$)$. The beneficial effect of these lower $P_{\text {prop }} / P_{\text {deac }}$ values on $Ð$ is, however, partly counteracted by the increase of $P_{\text {term }}$ (Figure 2e). The latter increase can be attributed to the as good as non-activated nature of termination and the limited importance on diffusional limitations on termination (Figure S2a; Supporting Information) at these low conversions. This similar control over $Ð$ at low conversions for the three cases is confirmed in Supporting Information (Figure S1a). At higher conversions both $P_{\text {prop }} / P_{\text {deac }}$ and $P_{\text {term }}$ (Figure 2c,d) become very similar for the three cases, but still the best control results for case 1 , since the suppression of the absolute termination rate is more pronounced 
(Figure S2b); Supporting Information). Hence, with a very dynamic temperature program, the lowest $Ð$ values are obtained at high conversions (Figure S1a); Supporting Information and Figure 2a,b).

\subsubsection{Variation of Monomer}

A second individual optimization pathway for ARGET ATRP of BMA is a fed-batch monomer addition program. The latter implies an increase of the reaction volume as a function of polymerization time. This optimization is performed at the maximal temperature of $90^{\circ} \mathrm{C}$, for illustration purposes only. Importantly, the term overall conversion $\left(X_{m, \text { overall }}\right)$ needs to be introduced [32]. This conversion is defined with respect to the initial molar amount of monomer used in the batch reference case $\left(n_{M, 0, \text { batch }}\right)$ :

$$
X_{m, \text { overall }}=\frac{n_{M, \text { reacted }}}{n_{M, 0, \text { batch }}}
$$

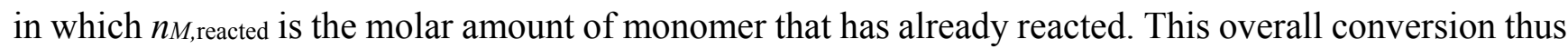
differs from the actual or in situ conversion $\left(X_{m}\right)$, which is defined with respect to amount of monomer added until the considered time. The overall conversion domain is divided into discrete subdomains of conversion increments of 0.125 until a final conversion of 0.75 is obtained as before. The addition profile is thus described by seven variables, each representing the added fraction with respect to the corresponding initial batch amount for a given conversion interval. This normalization with respect to the initial batch amount implies thus an implicit constraint for the selected amount per conversion interval and guarantees an unbiased comparison of the different sets of conditions considered. For the first variable, this is the initial amount present, which has a lower limit of $15 \mathrm{~mol} \%$ of the initial batch amount, whereas the other six variables represent the amount of monomer added in the interval. In each interval, a predefined amount of monomer is added in a continuous way per conversion increase of 0.0025 .

In Figure 3a, the Pareto-optimal front (green circles) is shown together with the batch reference case (red square), which clearly does not belong to this front, implying that an improvement of $Ð$ can be obtained with a simultaneous decrease in the polymerization time. Close inspection reveals that with a minimal increase of the polymerization time a large improvement of $\varnothing$ can be also accomplished, as long as $Ð$ values above a critical value of $c a$. 1.23 are targeted. Note that a decrease with $c a .0 .1$ for $Ð$ corresponds to an improvement of $20 \%$ of the standard deviation of the CLD. A lowering of the $Ð$ value below the critical value can be only obtained with a large penalty on the polymerization time, which can be clearly seen in the Pareto-optimal front displaying an L-shape.

Looking at the seven variables describing the monomer addition program (Figure $3 b$ ), it follows that more starved feed conditions at low overall conversions, i.e., lower monomer concentrations compared with the batch reference case, and the opposite conditions at high conversion are beneficial for control over chain length. This is shown in particular in Figure 4a which shows the change of the monomer concentration as a function of the overall monomer conversion for three illustrative cases of which the corresponding evolution of the cumulative molar fraction are depicted in Figure 3c. As for the temperature profile, case 1 clearly corresponds to the most dynamic case leading to the highest level of control over chain length. 
a)
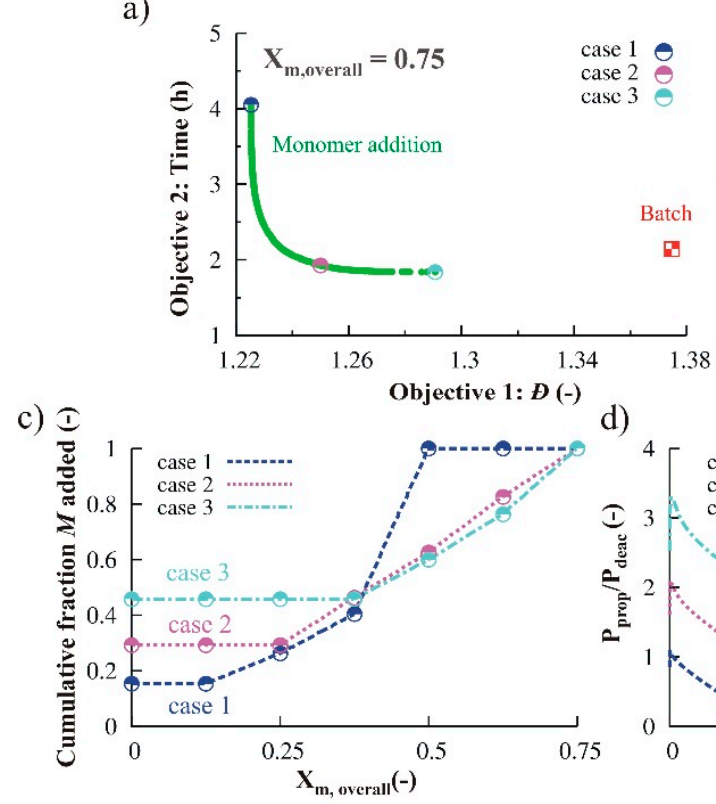

b)

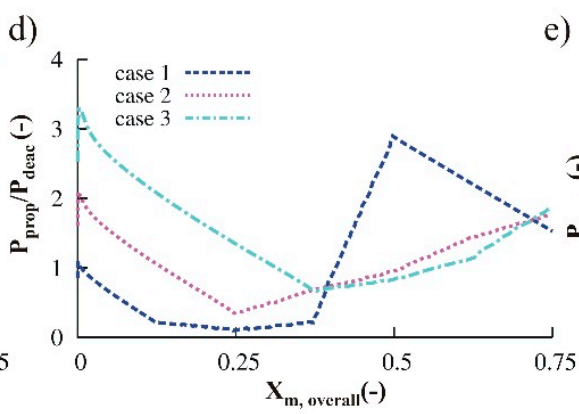

e)
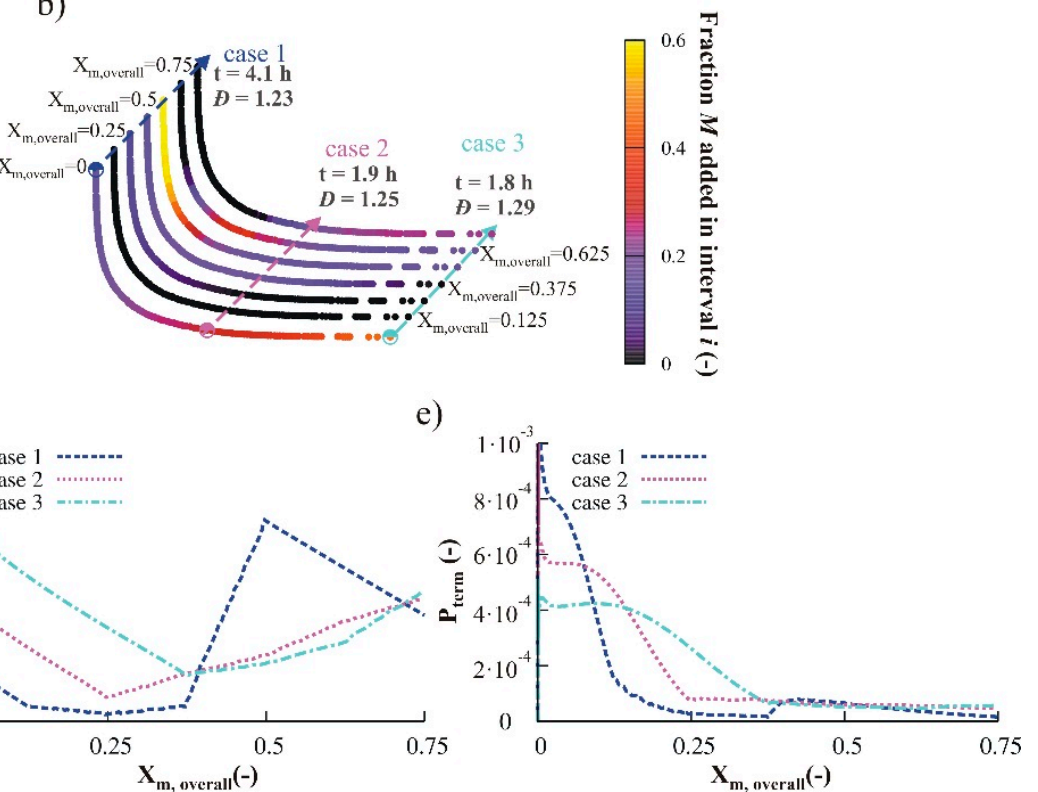

Figure 3. Results for the multi-objective optimization (MOO) of ARGET ATRP of BMA using a fed-batch monomer program (constant molar flow rate per $\Delta X_{m \text {,overall of }}$ 0.125 until $X_{m \text {,overall }}=0.75 ; 90{ }^{\circ} \mathrm{C} ;\left[R_{0} X\right]_{0} /\left[\mathrm{Cu}^{I I} X_{2 / L}\right]_{0} /\left[\mathrm{Sn}^{I I}(E H)_{2}\right]_{0}=1 / 0.005 / 0.05 ;$ batch case: $[M]_{0} /\left[R_{0} X\right]_{0}=200$ ). (a) Pareto-optimal front (green circles) and reference batch polymerization (red square); (b) Variation of the seven variables (fraction monomer added initially (variable 1) and during a conversion interval (variable 2-7) along the Pareto-optimal front; (c) Cumulative fraction of monomer added as a function of $X_{m \text {,overall }}$ for three selected cases in (a); (d) Ratio of probability for propagation over deactivation as a function of $X_{m \text {,overall }}$ for the selected cases; (e) Probability for termination as a function of $X_{m \text {,overall }}$ for the selected cases.

The differences between the three cases in both objectives (Figure 3a) can again be explained by looking at the evolution of $P_{\text {prop }} / P_{\text {deac }}$ (Figure $3 \mathrm{~d}$ ) and $P_{\text {term }}$ (Figure 3e). Note that the changes in reaction probabilities are now caused by differences in concentrations instead of differences in intrinsic/apparent rate coefficients. For low to intermediate overall conversions $\left(0.1<X_{m \text {,overall }}<0.4\right)$, very low $P_{\text {prop }} / P_{\text {deac }}$ values result, with the lowest ones for case 1, leading to a low $Ð$ for each case (Figure S1b; Supporting Information). This beneficial effect is, however, slightly counteracted at very low overall conversion $\left(X_{m, \text { overall }}<0.1\right)$ by higher $P_{\text {term }}$ values (Figure $3 \mathrm{e}$ ) since the radical concentration is also higher for a smaller reaction volume (Figure S3a; Supporting Information). On the other hand, the counteracting effect is of limited importance at higher overall conversions $\left(X_{m, \text { overall }}>0.1\right)$ taking into account that the in situ conversions are high (Figure S3b; Supporting Information) and thus already at low overall conversions the termination probability is strongly reduced. Hence, on an overall basis the positive effect on $P_{\text {prop }} / P_{\text {deac }}$ is dominant up to intermediate conversions, explaining the enhanced control with the fedbatch monomer addition program. Moreover, due to the excellent suppression of termination reactions, it is possible to increase the polymerization rate at higher overall conversions, with an acceptable disturbance of the control over chain length. 
a)

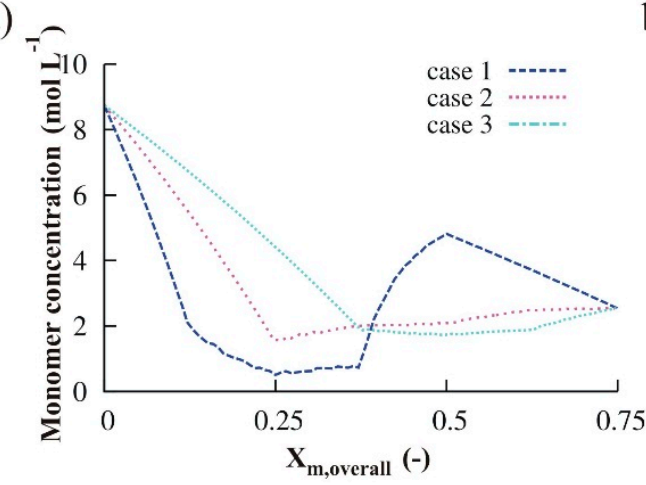

b)

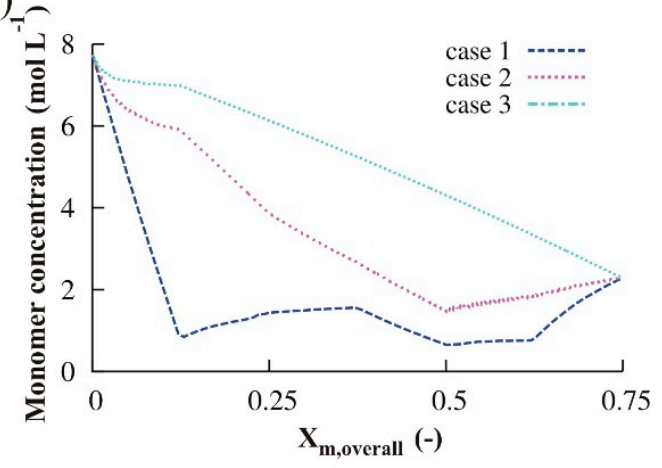

Figure 4. Monomer concentration as a function of overall conversion for the three cases in (a) the multi-objective optimization (MOO) of ARGET ATRP of BMA using a fed-batch monomer program (Figure 3) and (b) MOO of NMP of styrene using a fed-batch monomer program (Figure 8).

In particular for case 1 , which is characterized by the lowest $P_{\text {prop }} / P_{\text {deac }}$ and $P_{\text {term values it is afforded to }}$ add a large amount of monomer from intermediate overall conversions onwards $\left(X_{m, \text { overall }}>0.4\right)$. This only leads to a limited increase of $P_{\text {prop }} / P_{\text {deac }}$ and a very limited increase of $P_{\text {term }}$ The latter probability is only slightly increased since the large decrease of the radical concentration due to dilution compensates the corresponding increase of the apparent termination reactivity (Figure S3a,b; Supporting Information). On the other hand, it should be reminded that a lower radical concentration leads to a lower propagation rate and thus higher polymerization times, consistent with the L-shape of the Pareto-optimal front.

\subsubsection{Variation of Reducing Agent}

A final individual optimization pathway that is explored for the ARGET ATRP case study is the fed-batch addition of reducing agent. The total amount of reducing agent added is taken equal to the batch amount and is the same as used in the aforementioned simulations. Again a constant temperature of $90{ }^{\circ} \mathrm{C}$ is chosen. The addition profile is described by seven variables, again per conversion increment of 0.125 . The first variable represents the fraction of the initial batch amount present at the start of the ARGET ATRP. The six remaining variables are the fractions of the initial batch amount added during an interval in a continuous manner at a constant molar flow rate per conversion increase of 0.0025 .

The results (Figure 5a) show that also in this case a trade-off between both objectives results in an L-shaped Pareto-optimal front. The values of the seven variables, along the Pareto-optimal front, are shown in Figure 5b. From this figure it can be concluded that the fastest polymerization time is equal to the batch case. A lower initial fraction of reducing agent leads to a higher polymerization time, but lower $Ð$. Throughout the remainder of the polymerization, smaller feed rates at low to intermediate conversion also lead to longer polymerization times and lower dispersities, whereas feeding the reducing agent at low conversions results in a faster polymerization but higher $\bigoplus$. 
a)

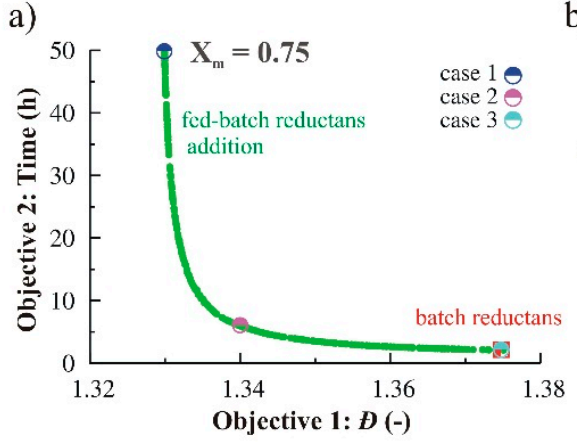

b)

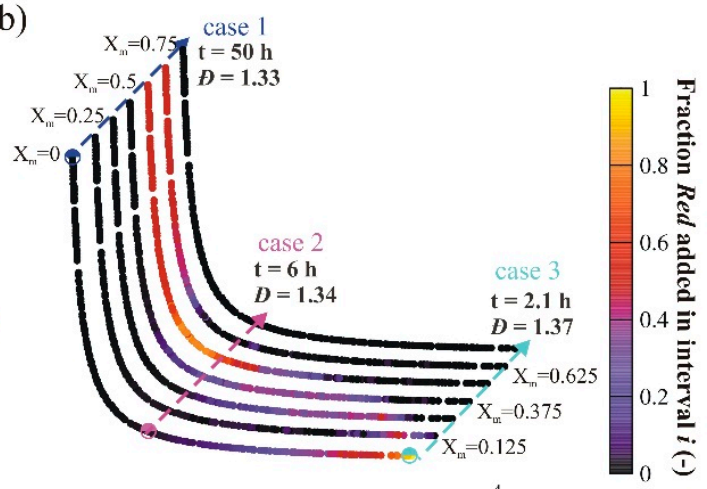

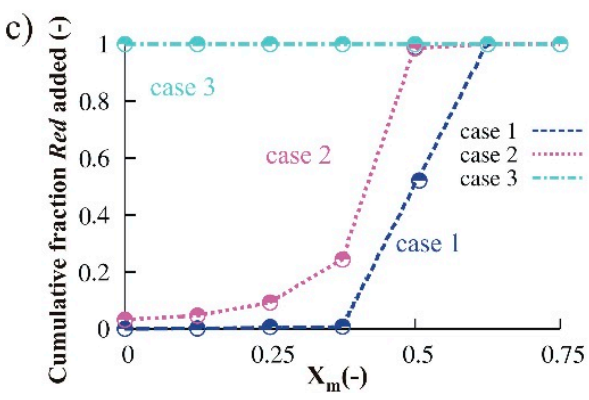

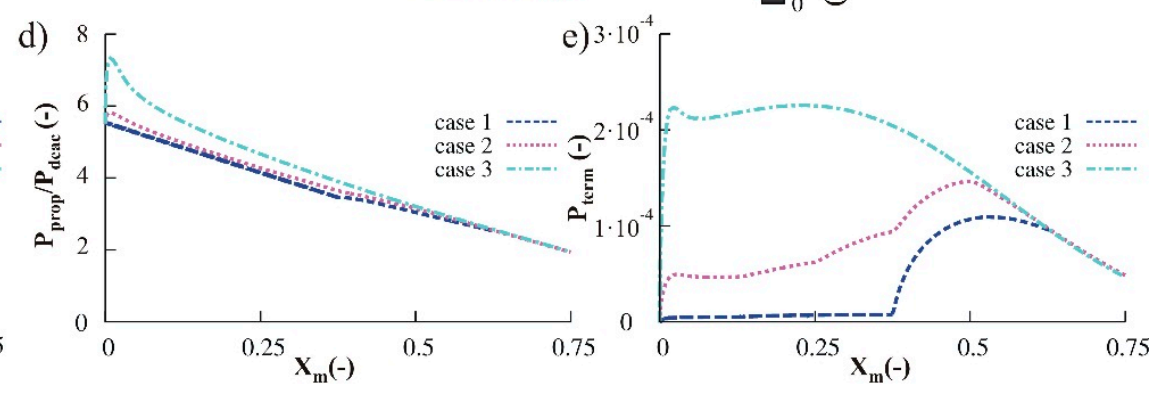

Figure 5. Results for the multi-objective optimization (MOO) of ARGET ATRP of MBA using a fed-batch reducing agent program (constant molar flow rate per $\Delta X_{m}=0.125$ until $X_{m}=0.75 ;[M]_{0} /\left[R_{0} X\right]_{0} /\left[C u^{I I} X_{2} / L\right]_{0}=200 / 1 / 0.005 ; 90{ }^{\circ} \mathrm{C}$ : batch case: $\left.\left[R_{0} X\right]_{0} /\left[\operatorname{Sn}^{I I}(E H)_{2}\right]_{0}=0.05\right)$. (a) Pareto-optimal front (green circles) and reference batch case (red square); (b) Variation of the seven variables (fraction reducing agent added initially (variable 1) and during a certain conversion interval (variable 2-6)) along the Pareto-optimal front; (c) Cumulative fraction of reducing agent added as a function of $X_{m}$ for three selected cases in (a); (d) Ratio of probability of propagation to deactivation as a function of $X_{m}$ for the selected cases; (e) Probability for termination as a function of $X_{m}$ for the selected cases.

The feeding profiles as a function of conversion for three illustrative cases are shown in Figure 5c. To explain the variation in the objectives the reaction probabilities are shown in Figure 5e,d. Looking at $P_{\text {prop }} / P_{\text {deac }}$ (Figure $5 \mathrm{~d}$ ), it can be seen that very limited differences can be observed, this in contrast to the application of a temperature and fed-batch monomer program. On the other hand, Figure 5e shows that for case 1 (long polymerization time, low $Đ$; Figure S1c; Supporting Information) $P_{\text {term }}$ is practically zero for conversions lower than 0.5 and thus a good control over chain length can be obtained with a fed-batch addition of reducing agent. This is due to a very low radical concentration (Figure S4; Supporting Information) since the reducing agent, needed for the reduction of a deactivator species, is only brought into the reaction mixture very slowly. This logically also leads to a decrease of the polymerization rate.

It can be concluded that the underlying reason of the examined individual optimization pathways can be different, as they can affect the reaction rates differently. Hence, additional improvement can be expected for specific combinations of individual optimization paths. This conjecture is explored in the next subsection. 


\subsubsection{Simultaneous Variation of Different Process Conditions}

In this subsection, a comparison between the different individual optimization pathways and combinations of these individual pathways are explored. The results of the individual optimizations and only the combinations leading to an improvement of the Pareto-optimal front (with respect to the individual optimizations) are shown in Figure 6.

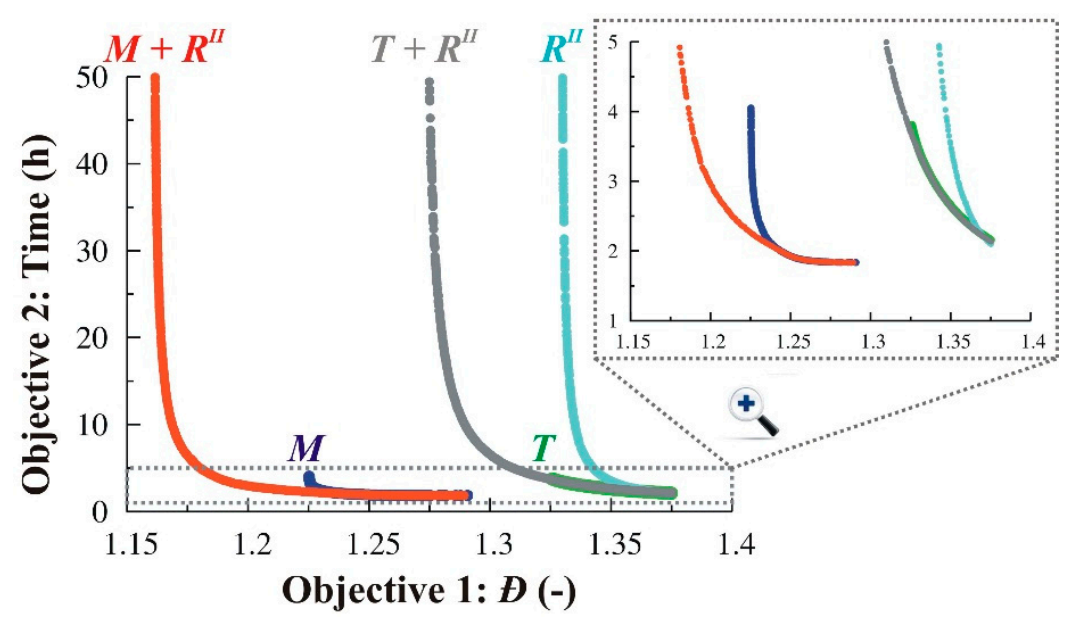

Figure 6. Comparison between multi-objective optimization (MOO) results of the different individual optimization pathways: fed-batch addition of reducing agent $\left(R^{I I}\right)$ (teal; Figure 5a), temperature program $(T)$ (green; Figure 2a), fed-batch addition of monomer $(M)$ (blue; Figure $3 a)$, the synergetic combination of temperature program and fed-batch addition of reducing agent (grey), and the synergetic combination of fed-batch addition of monomer and reducing agent (red).

This figure shows that applying a fed-batch monomer program results in a Pareto-optimal front that dominates the Pareto-optimal fronts of the other two individual pathways, i.e., applying a temperature program and fed-batch reducing agent program. Only two combinations lead to a synergetic effect. A first one is the combination of a fed-batch reducing agent program with a temperature program. An even more synergetic pathway is the combination of a fed-batch monomer and reducing agent addition program. In other words, combination of a temperature program with a fed-batch monomer program and combinations of all three do not result in a significant improvement of the Pareto-optimal front.

To understand the improvement of the two synergetic cases, it is necessary to fully understand the differences of the individual optimization pathways, as previously discussed. For the temperature and the fed-batch monomer program, it was explained that both pathways lead at lower (overall) conversions to a lowered $P_{\text {prop }} / P_{\text {deac }}$, i.e., to a decrease in the average number of monomer units added per activation-growth-deactivation cycle. However, the beneficial effect on the $\oslash$ profiles was partly diminished due to a higher $P_{\text {term. }}$. Applying a fed-batch reducing agent program leads on the other hand to a reduction of $P_{\text {term }}$ at lower conversion, but hardly influences $P_{\text {prop }} / P_{\text {deac. }}$. Hence, it can be understood that combining the latter with a temperature or fed-batch monomer program leads to an improved Pareto-optimal front. In contrast, combining a temperature and fed-batch monomer program only results 
in a very limited improvement since both rely on suppressing/favoring the same reactions, which is also confirmed by the MOO.

\subsection{Multi-Objective Optimization of NMP}

The second case study involves the MOO of NMP of styrene initiated by MAMA-SG1 to optimize the process with respect to polymerization time, livingness and dispersity, i.e., three objectives are now considered. Several individual optimization pathways are again explored, namely optimization via a temperature program, fed-batch monomer addition, and fed-batch addition of free nitroxide, the persistent radical. Also the combination of optimization pathways is again examined. As before, for all optimizations considered, a constraint on the polymerization time of maximum $50 \mathrm{~h}$ is imposed. The conditions, $[M]_{0} /\left[R_{0} X\right]_{0}=1000 / 1\left(120^{\circ} \mathrm{C}\right)$, are taken as batch reference conditions.

\subsubsection{Variation of Temperature}

As a first optimization pathway the use of a temperature program is again investigated. The temperature program is described in the same way as with the MOO of ARGET ATRP. The seven variables are now allowed to vary between 80 and $120^{\circ} \mathrm{C}$, based on literature data for the isothermal batch NMP of styrene [61]. The corresponding results are shown in Figure 7, considering three objectives, namely time, $\doteq$, and EGF. For completeness it is mentioned here that a quasi-identical Pareto-optimal front is obtained in case only two objectives are selected (e.g., time and D; Figure S5; Supporting Information). However, for decision making toward a preferred operation point it is more suited to focus on the MOO case with three objectives (see Figure 7a), taking into account that the EGF now varies significantly along the Pareto-optimal front. For isothermal NMP, the Pareto-optimal front is also shown in Figure 7a. Clearly, this front is not a subset of the Pareto-optimal front of the temperature program optimization. This means that a non-isothermal temperature program is always beneficial compared to the classical isothermal case.

From Figure $7 \mathrm{~b}$, which shows the variation of the temperature at the seven distinct conversions, it can be concluded that to find optimal trade-offs between the three objectives, a more complex temperature profile should be applied. This is also confirmed in Figure 7c, which displays the actual change of the temperature with conversion for three illustrative cases. The cases differ in the extent of their non-isothermicity but all start with a lower polymerization temperature. For the third case, which results in the lowest batch time but leads to the highest $Ð$ and lowest EGF, the temperature is maintained at the upper limit temperature throughout the major part of the polymerization and thus the isothermal case of $120{ }^{\circ} \mathrm{C}$ is mimicked to a certain extent. In contrast, for the other two cases, which lead to a higher control over the NMP, the temperature is initially at its lower limit value, after which it is increased until a conversion of 0.125 is reached. Hereafter the temperature is lowered until a conversion of 0.25 results. Only at high conversion $\left(X_{m}>0.60\right)$ the temperature is significantly increased toward the maximum value and thus a strongly non-isothermal temperature profiles is established, in particular for the first case. 
a)
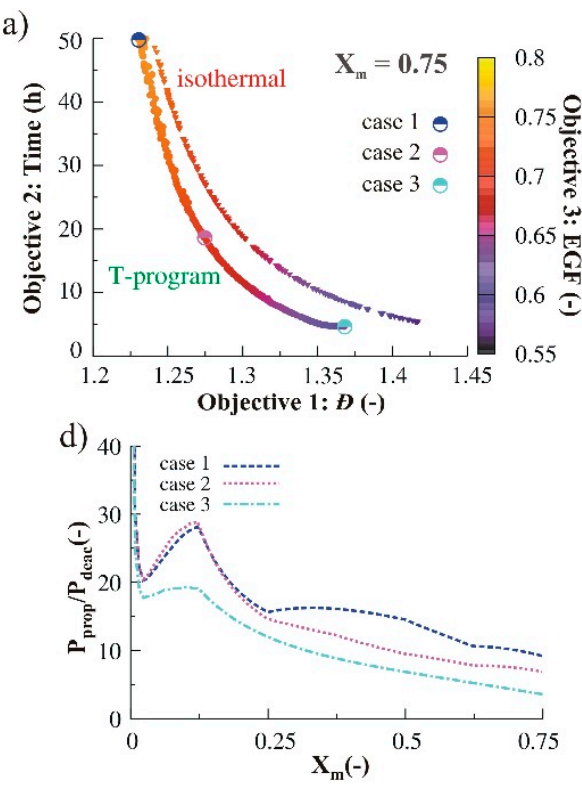

b)

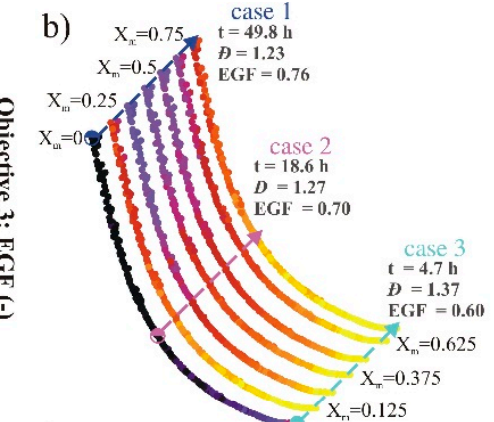

\section{。}

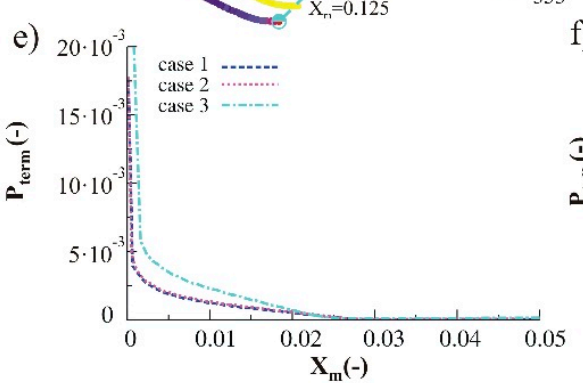

c)
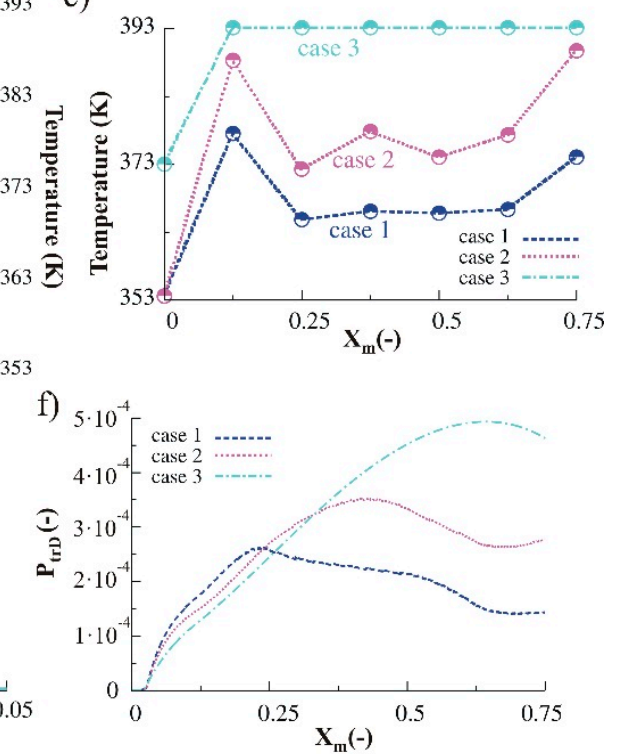

Figure 7. Results for the multi-objective optimization (MOO) of NMP of styrene using a piecewise linear temperature program $\left(\Delta X_{m}=0.125\right.$ until $\left.X_{m}=0.75 ;[M]_{0} /\left[R_{0} X\right]_{0}=1000 / 1\right)$. (a) Pareto-optimal front and corresponding isothermal front; (b) Variation of the temperature at the interval boundaries along the Pareto-optimal front; (c) Temperature as a function of $X_{m}$ for three selected cases; (d) Ratio of probability for propagation over deactivation as a function of $X_{m}$ for the selected cases; (e) Probability for termination; (f) Probability for transfer to dimer as a function of $X_{m}$.

These at first sight counterintuitive trends in the temperature programs can be rationalized by considering the relative changes of the key reaction probabilities. As with the ARGET ATRP case, $P_{\text {prop }} / P_{\text {deac }}$ (Figure $7 \mathrm{~d}$ ) as well as $P_{\text {term }}$ (Figure 7e) play an important role in the obtained microstructural control. As explained above, the lower $P_{\text {prop }} / P_{\text {deac, }}$ the smaller the average number of monomer units incorporated per activation-growth-deactivation cycle and, hence, the lower the $\oslash$ value. A higher $P_{\text {term }}$ leads to an increase in $Ð$ but also to a lowering of EGF. In the case of the NMP of styrene, the reaction probability for chain transfer to dimer ( $P_{t r D}$; Figure $7 \mathrm{f}$ ) needs also to be considered, specifically at elevated temperature $[62,74]$.

At low conversion, the beneficial effect of starting with a lower temperature has already been explained in Fierens et al. [61] in which an isothermal NMP $\left(120{ }^{\circ} \mathrm{C}\right)$ was compared with a NMP conducted using a stepwise temperature profile with a lower initial temperature aiming to obtain a conversion of $X_{m}$ of 0.75 within the same polymerization time as the isothermal case. With the stepwise profile less termination is occurring in the early stage of the NMP, leading to a less dramatic manifestation of the persistent radical effect. This is confirmed in Figure $7 \mathrm{e}$, in which $P_{\text {term }}$ is clearly lowered and thus EGF is increased when starting at a lower initial temperature (decreasing temperature for case 3, 2 and 1). On the other hand, at these low conversions, $P_{\text {prop }} / P_{\text {deac }}$ and $P_{t r D}$ are higher compared to the quasi-isothermal case 3, leading to higher initial $D$ values (Figure S6a; Supporting Information) for case 1 and 2. From intermediate conversions onwards, in contrast, lowering the polymerization temperature is beneficial for the evolution of $\oslash$ and EGF. This can be explained by looking at the pronounced change of $P_{t r D}$ (Figure $7 \mathrm{f}$ ). The lower temperature in cases 2 and 3 for intermediate 
conversions suppresses the influence of the chain transfer to dimer, leading to the observed better control over $\oslash$ and EGF. On the one hand, less dimer is formed via thermal auto-initiation and on the other hand chain transfer to dimer is suppressed, taking into account its relatively high activation energy $\left(28 \mathrm{~kJ} \cdot \mathrm{mol}^{-1}\right.$ in Table 1). This, obviously, also leads to a decreased polymerization rate. Note that the increase of the temperature at the higher conversions (Figure 7c) does not result in a severe penalty on the relevance of chain transfer to dimer (Figure 7f) since the dimer concentration (Figure S7a; Supporting Information) is already significantly decreased. This temperature increase will increase the polymerization rate. However, still a relatively slow NMP is obtained on an overall basis if a dynamic temperature profile is considered.

\subsubsection{Monomer Addition}

A second optimization pathway for NMP of styrene is the fed-batch addition of monomer. A constant temperature of $120{ }^{\circ} \mathrm{C}$ is considered for illustration purposes. Again the overall conversion domain is divided into discrete subdomains of conversion increments of 0.125 . The addition profile is again described by seven variables, each representing the added fraction with respect to the corresponding initial batch amount. The initial fraction present has now a lower limit of $10 \mathrm{~mol} \%$. Note that this monomer addition approach can be seen as an extension of the work of Bentein et al. [62] in which fixed small amounts of monomer were added for the NMP of styrene, using phenylethyl-SG1 as NMP initiator.

As can be seen in Figure 8a, fed-batch monomer addition results in an improvement compared to the batch case. Looking at the value of each variable along the Pareto-optimal front (Figure 8b) some trends can be observed. It is beneficial to start the NMP with a low amount of monomer after which the remainder of the initial batch amount monomer is added continuously. As for the temperature variation, a more complex profile allows a higher level of control. Selecting again three illustrative cases, this becomes clearer when considering the explicit variation of the cumulative amount of monomer added with overall conversion (Figure 8c). The corresponding monomer concentration profiles are provided in Figure 4b. For case 3, the total amount of monomer is already added in the first interval, leading to a high monomer concentration and a high polymerization rate but also to a higher $\oslash$ and a lower EGF. For the other two cases, the addition of the remainder monomer amount occurs throughout the whole NMP. With the most starved-feed monomer conditions (case 1) the highest degree of control is obtained despite an increase in polymerization time.

At low conversions, the beneficial effect of starting with a low initial amount of monomer can be partially linked to $P_{\text {prop }} / P_{\text {deac }}$ (Figure $8 \mathrm{~d}$ ). This ratio is the lowest for case 1 where on average a lower amount of monomer is incorporated in each activation-growth-deactivation cycle, already from low conversion onwards. In other words, the initial spike in the $Ð$ profile (Figure S6b; Supporting Information) is lower and $Ð$ declines to a lower value since controlled conditions are already obtained at a lower conversion. Additionally, the improved control can be related to the positive evolution of $P_{\text {term }}$ (Figure 8e) and $P_{t r D}$ (Figure 8f). It is important to mention that case 1 has the highest living macroradical concentration of the three cases considered. Nevertheless, the rate of termination is the lowest since termination reactions are more diffusion controlled throughout the NMP. In case 1, the in situ conversion is namely very high $(\approx 0.9)$ for the largest part of the polymerization (Figure S8a; Supporting Information) and diffusional limitations are more pronounced, explaining the lower $P_{\text {term }}$ values. 
The reduced importance of chain transfer to dimer can be understood by looking closely at the dimer formation in which two monomer molecules need to undergo a Diels-Alder reaction. If a fed-batch monomer addition program is applied, especially under starved feed conditions, i.e., cases 1 and 2, the monomer concentration is kept low (Figure $4 \mathrm{~b}$ ), thereby strongly reducing the dimer concentration (Figure S8b; Supporting Information) and thus also lowering $P_{t r D}$.
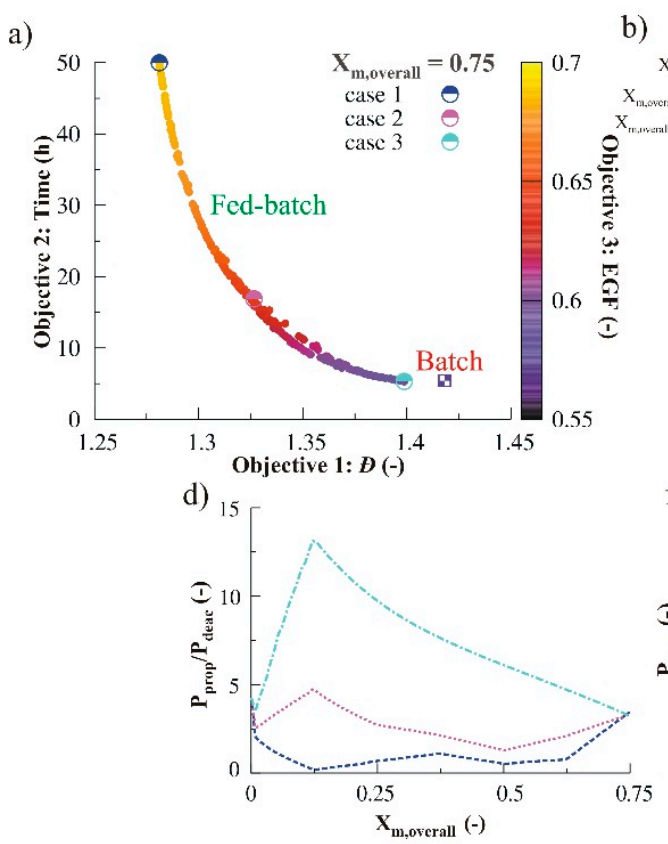

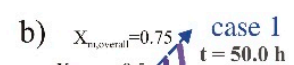

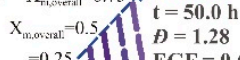

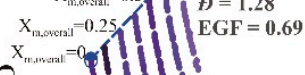

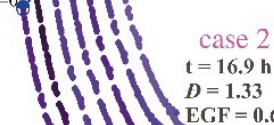

110 the $=0.64$ case 3
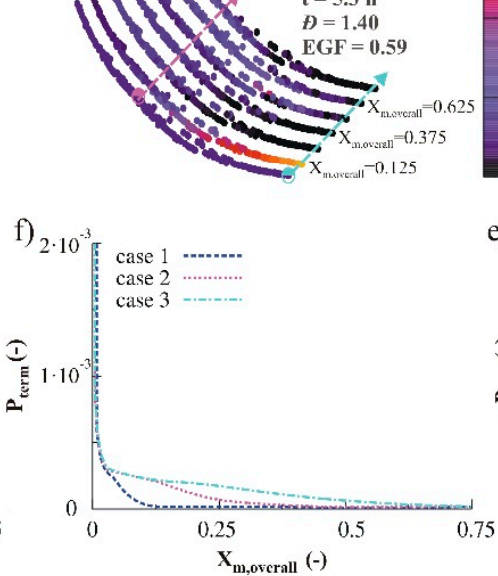

c)
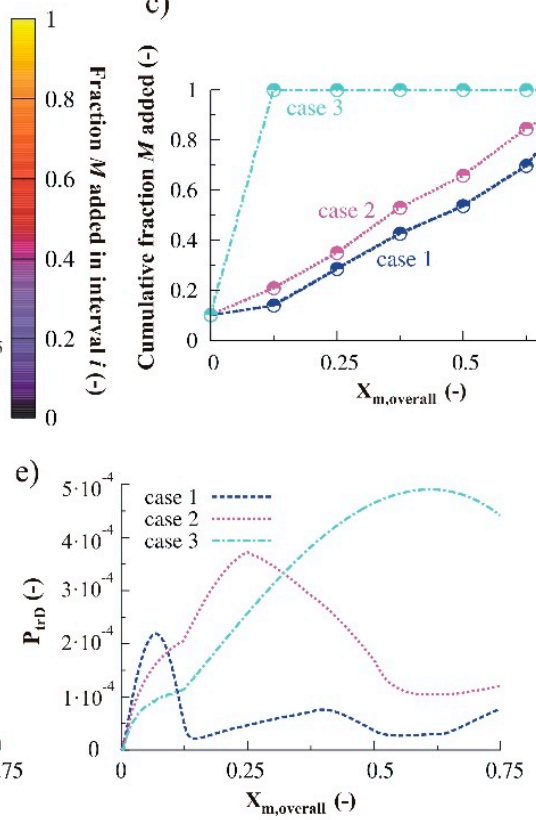

Figure 8. Results for the multi-objective optimization (MOO) of NMP of styrene $\left(120{ }^{\circ} \mathrm{C}\right)$ using a fed-batch monomer program (constant molar flow rate per $\Delta X_{m \text {,overall of } 0.125 \text { until }}$ $\left.X_{m, \text { overall }}=0.75 ;[M]_{0} /\left[R_{0} X\right]_{0}=1000 / 1\right)$. (a) Pareto-optimal front and corresponding isothermal front; (b) Variation of the seven variables (fraction monomer added initially (variable 1) and during a conversion interval (variable 2-7) along the Pareto-optimal front; (c) Cumulative fraction of monomer added as a function of $X_{m \text {,overall }}$ for three selected cases; (d) Ratio of probability for propagation to deactivation as a function of $X_{m \text {,overall }}$ for the selected cases; (e) Probability for termination as a function of $X_{m \text {,overall }}$ for the selected cases;

(f) Probability for chain transfer to dimer as a function of $X_{m, \text { overall }}$ for the selected cases.

\subsubsection{Variation of Initial Nitroxide Loading}

The beneficial influence of an excess of nitroxide with respect to NMP initiator, has already been discussed in literature $[39,44,75]$. Therefore, as a third possibility to optimize the NMP characteristics, the addition of nitroxide at the start of the polymerization is investigated. To stay within representative NMP conditions, the maximum initial ratio of nitroxide $(X)$ to NMP initiator $\left(R_{0} X\right)$ has been limited to 0.5 . Again a constant polymerization temperature of $120{ }^{\circ} \mathrm{C}$ is selected. For completeness it is mentioned here that also a MOO was performed with a nitroxide fed-batch addition program. However, this optimization revealed that the Pareto-optimal front only consists of points in which the total amount of nitroxide is added at the start of the polymerization, explaining why the initial loading was finally taken up as the only variable. 
The Pareto-optimal front considering three objectives (time, $Ð$, and EGF) is given in Figure 9a. The corresponding variation of the initial molar ratio of nitroxide to NMP initiator is provided in Figure 9b. When an excess of nitroxide is initially present, termination is already strongly suppressed from the start of the NMP and almost no build-up of nitroxide is needed to create a sufficient high concentration of nitroxide species (Figure 9d). This can also be inferred from Figure $9 \mathrm{c}$ in which $P_{\text {prop }} / P_{\text {deac }}$ is plotted as a function of conversion. At the initial stage, excess nitroxide leads to a sharp decrease in this ratio, since deactivation reactions are favored from the beginning of the polymerization, and thus the $Ð$ profile falls back to a much lower value from an early stage in the polymerization (Figure S6c; Supporting Information). The beneficial effect is, however, counteracted at higher conversions by the higher importance of the chain transfer to dimer reaction (Figure 9e) leading to a rise in the $D$ profile as a function of conversion. This higher importance can be related to the higher impact of radical generation via thermal initiation (Figure S9a; Supporting Information), since a lower polymerization rate allows an increase of the dimer concentration (Figure S9b); Supporting Information) during a significant period. Hence, the concentration of both reactants for chain transfer to dimer increases in such way that $P_{t r D}$ increases, explaining the observed $Ð$ profiles.

a)

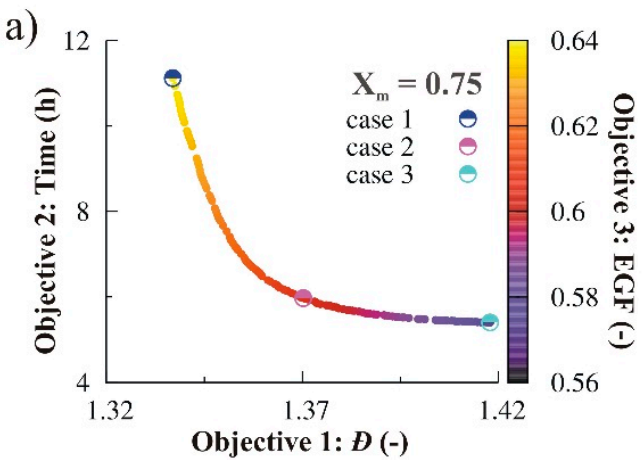

c)

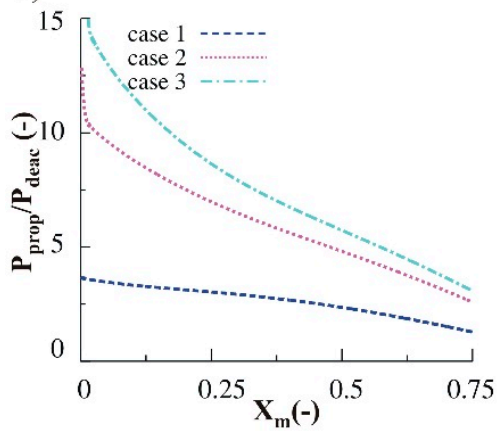

d)

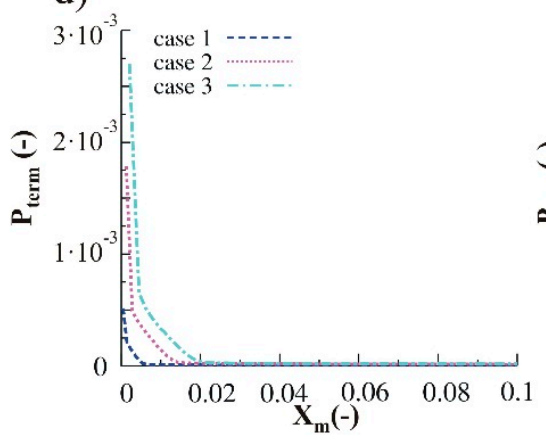

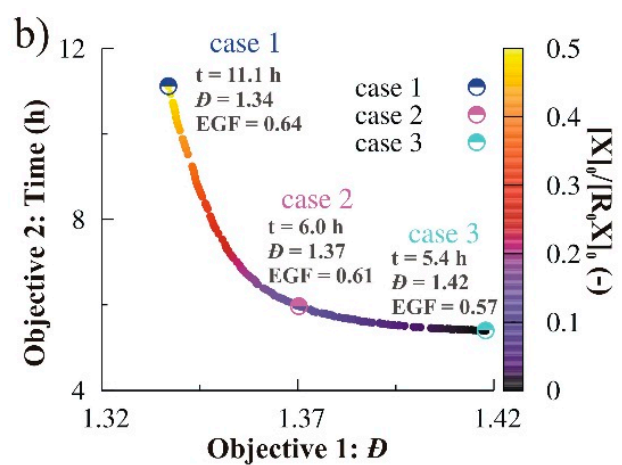

e)

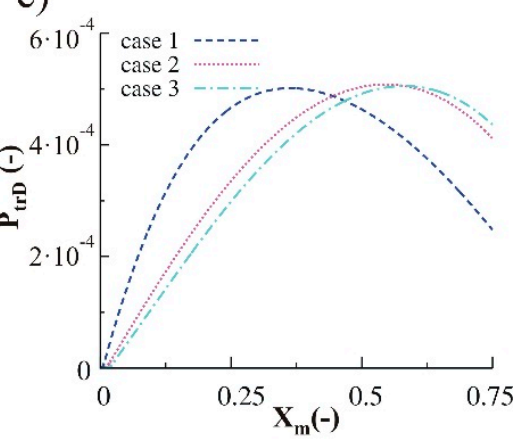

Figure 9. Results for the multi-objective optimization (MOO) of NMP of styrene $\left(120{ }^{\circ} \mathrm{C}\right)$ using excess initial nitroxide $\left(\Delta X_{m}=0.125\right.$ until $X_{m}=0.75 ;\left([M]_{0} /\left[R_{0} X\right]_{0}=1000 / 1\right)$. (a) Pareto-optimal front and corresponding isothermal front; (b) Variation of $[X]_{0} /\left[R_{0} X\right]_{0}$ along the Pareto-optimal front; (c) Ratio of probability for propagation to deactivation as a function of conversion for the selected cases; (d) Probability for termination as a function of $X_{m}$ for the selected cases; (e) Probability for chain transfer to dimer as a function of $X_{m}$ for the selected cases. 
It should be stressed that overall still a large improvement of the $\oslash$ can be obtained with a relative small penalty in polymerization time, as witnessed from the L-shape of the Pareto-optimal front (Figure 9a). This result is corroborated by the reported observation that a small addition of nitroxide $(<10 \mathrm{~mol} \%)$ is beneficial for NMP processes in general, and that higher amounts decrease the polymerization rate excessively [39].

\subsubsection{Simultaneous Variation of Different Process Conditions}

In a final step, combinations of the aforementioned individual pathways are explored, considering the most complex case of the three objectives time, $Ð$ and EGF. The most interesting combinations of these advanced MOO simulations are shown in Figure 10, accompanied by the MOO fronts obtained via a single variation. To avoid misinterpretation, the Pareto optimal front is depicted twice using the time as the common objective. Looking only at the result of the individual optimization pathways it can be seen that applying a temperature program can be considered the best option with respect to all three objectives.
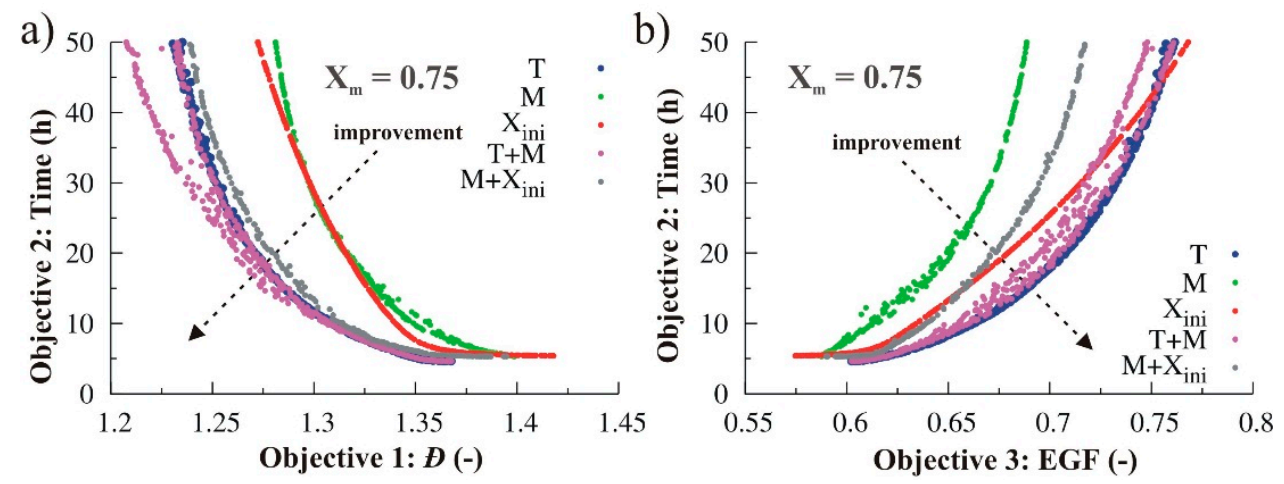

Figure 10. Comparison between multi-objective optimization (MOO) results of NMP of styrene for the different individual optimization pathways: temperature program $(T)$ (blue; Figure 7a), fed-batch addition of monomer $(M)$ (green; Figure $8 \mathrm{a}$ ), excess initial nitroxide $\left(X_{\text {ini }}\right)$ (red; Figure 9a), the synergetic combination of temperature program and fed-batch monomer addition (purple), the synergetic combination of fed-batch addition of monomer and excess initial nitroxide (grey) (a) projection for objective $1(\bigoplus)$ and objective 2 (time), and (b) projection for objective 3 (EGF) and objective 2 (time).

Further inspection shows that combination of a fed-batch monomer addition and an initial excess amount of nitroxide (yellow dots) is strongly synergetic, leading to a much improved Pareto-optimal front compared to the individual cases (green and red circles), especially for $Ð$ (Figure 4a). This synergetic effect is due to positive effect of the initial presence of the free nitroxide which leads to a strongly decreased $P_{\text {prop }} / P_{\text {deac }}$ and thus a lower $D$ from low conversions onwards. Moreover, fed-batch addition of monomer additionally allows to suppress chain transfer to dimer reactions, which cannot be achieved if the nitroxide loading is the only variable, as explained in the previous subsection.

Finally, it follows that the combination of a temperature profile with a fed-batch monomer addition (purple dots) leads to Pareto-optimal fronts that cannot be represented by a single line, taking into account the $2 \mathrm{D}$ representation. This is thus a direct consequence of the definition of Pareto-optimality in which only improvement in one objective is needed, i.e., the simultaneous improvement of both EGF 
and $Ð$ is not strictly demanded. Hence, depending on the actual RDRP product requirements, it follows that a certain combination will be seen as suitable are not.

\section{Conclusions}

For the first time, Pareto-optimal fronts have been determined for two important reversible deactivation radical polymerization (RDRP) techniques, i.e., activators regenerated electron transfer atom transfer radical polymerization (ARGET ATRP) and nitroxide-mediated polymerization (NMP). For the simulations, the NSGA-II algorithm has been successfully implemented and combined with literature kinetic models. Diffusional limitations are accounted for on termination using a composite $k_{t}$-model. As design variables the temperature and molar amounts of the involved species have been considered per conversion interval of 0.125 , until a conversion of 0.75 is reached. A distinction is made between 2-dimensional multi-objective optimization (MOO) for ARGET ATRP of BMA with as objectives time and $\oslash$ and 3-dimensional MOO for NMP of styrene with as objectives time, $\oslash$, and EGF.

In both RDRPs, the conditions belonging to the Pareto-optimal front perform better than the corresponding isothermal or batch conditions. In particular, significant gain in time is obtained for a given control over the RDRP. A typical Pareto-optimal front is L-shaped, implying that control can be improved without a significant penalty on time up to a critical $Ð$.

For ARGET ATRP of BMA, the strongest improvement for individual optimization pathways is obtained if a fed-batch addition of monomer is performed. Further improvement is obtained in case a combination of two optimization pathways is allowed. The most promising results are obtained when either a temperate program is combined with a fed-batch addition of reducing agent or a fed-batch addition of monomer is combined with a fed-batch addition of reducing agent. This can be explained by analyzing the reaction probabilities for the radical species. Fed-batch addition of a reducing agent allows lowering the termination reaction probability, whereas fed-batch addition of monomer and a temperature program allow decreasing the propagation to deactivation reaction probability, which ensures a more controlled incorporation of monomer units per activation-growth-deactivation cycle on an average basis.

For NMP of styrene, considering individual optimization paths, a temperature program is the most suited, as it allows to suppress termination at low conversion, to decrease the propagation to deactivation reaction probability and to avoid a dominant occurrence of chain transfer to dimer at high conversion. A combination with fed-batch addition of monomer or a non-zero initial deactivator concentration allows further improvement. The former combination does not, however, automatically imply a simultaneous improvement in EGF and $Đ$.

Finally, it should be stressed that although NMP and ARGET ATRP are vastly different, it has been demonstrated that the road to maximize their potential consists of the common themes of optimizing the RDRP initiation process, mediating the activation/growth/deactivation cycles and suppressing termination and chain transfer reactions.

\section{Acknowledgments}

The authors acknowledge financial support from the Long Term Structural Methusalem Funding by the Flemish Government, the Interuniversity Attraction Poles Programme-Belgian State-Belgian Science 
Policy, and the Fund for Scientific Research Flanders (FWO; G.0065.13N). Dagmar R. D'hooge acknowledges the Fund for Scientific Research Flanders (FWO) through a postdoctoral fellowship.

\section{Supplementary Information}

Supplementary Information can be accessed at http://www.mdpi.com/2073-4360/7/4/0655/s1.

\section{Author Contributions}

All authors have contributed to the writing of the results and discussion. Stijn K. Fierens has performed the simulations.

\section{Conflicts of Interest}

The authors declare no conflict of interest.

\section{References}

1. Braunecker, W.A.; Matyjaszewski, K. Controlled/living radical polymerization: Features, developments, and perspectives. Progr. Polym. Sci. 2007, 32, 93-146.

2. Matyjaszewski, K. Handbook of Radical Polymerization; Wiley: Hoboken, NJ, USA, 2002.

3. Chiefari, J.; Chong, Y.K.; Ercole, F.; Krstina, J.; Jeffery, J.; le, T.P.T.; Mayadunne, R.T.A.; Meijs, G.F.; Moad, C.L.; Moad, G.; et al. Living free-radical polymerization by reversible addition-fragmentation chain transfer: The raft process. Macromolecules 1998, 31, 5559-5562.

4. Chong, Y.K.; Le, T.P.T.; Moad, G.; Rizzardo, E.; Thang, S.H. A more versatile route to block copolymers and other polymers of complex architecture by living radical polymerization: The raft process. Macromolecules 1999, 32, 2071-2074.

5. Moad, G.; Chiefari, J.; Chong, Y.K.; Krstina, J.; Mayadunne, R.T.A.; Postma, A.; Rizzardo, E.; Thang, S.H. Living free radical polymerization with reversible addition-fragmentation chain transfer (the life of raft). Polym. Int. 2000, 49, 993-1001.

6. D'hooge, D.R.; Vachaudez, M.; Stadler, F.J.; Reyniers, M.-F.; Coulembier, O.; Bailly, C.; Dubois, P.; Marin, G.B. Assessment of end-group functionality in atom transfer radical polymerization of $N$-isopropylacrylamide. Eur. Polym. J. 2013, 49, 2344-2355.

7. Metz, N.; Theato, P. Controlled synthesis of poly(acetone oxime acrylate) as a new reactive polymer: Stimuli-responsive reactive copolymers. Eur. Polym. J. 2007, 43, 1202-1209.

8. Ferrari, R.; Yu, Y.; Morbidelli, M.; Hutchinson, R.A.; Moscatelli, D. $\varepsilon$-Caprolactone-based macromonomers suitable for biodegradable nanoparticles synthesis through free radical polymerization. Macromolecules 2011, 44, 9205-9212.

9. Wang, W.; Hutchinson, R.A. PLP/SEC/NMR study of free radical copolymerization of styrene and glycidyl methacrylate. Macromolecules 2008, 41, 9011-9018.

10. Wang, J.-S.; Matyjaszewski, K. Controlled/“living” radical polymerization. Atom transfer radical polymerization in the presence of transition-metal complexes. J. Am. Chem. Soc. 1995, 117, 5614-5615. 
11. Patten, T.E.; Matyjaszewski, K. Atom transfer radical polymerization and the synthesis of polymeric materials. Adv. Mater. 1998, 10, 901-915.

12. Matyjaszewski, K.; Xia, J. Atom transfer radical polymerization. Chem. Rev. 2001, 101, 2921-2990.

13. Vachaudez, M.; D’hooge, D.R.; Socka, M.; Libiszowski, J.; Coulembier, O.; Reyniers, M.F.; Duda, A.; Marin, G.B.; Dubois, P. Inverse dependencies on the polymerization rate in atom transfer radical polymerization of $\mathrm{N}$-isopropylacrylamide in aqueous medium. React. Funct. Polym. 2013, 73, 484-491.

14. Teodorescu, M.; Matyjaszewski, K. Controlled polymerization of (meth)acrylamides by atom transfer radical polymerization. Macromol. Rapid Commun. 2000, 21, 190-194.

15. Lutz, J.F.; Matyjaszewski, K. Kinetic modeling of the chain-end functionality in atom transfer radical polymerization. Macromol. Chem. Phys. 2002, 203, 1385-1395.

16. Ballard, N.; Salsamendi, M.; Santos, J.I.; Ruipérez, F.; Leiza, J.R.; Asua, J.M. Experimental evidence shedding light on the origin of the reduction of branching of acrylates in ATRP. Macromolecules 2014, 47, 964-972.

17. Nicolas, J.; Guillaneuf, Y.; Lefay, C.; Bertin, D.; Gigmes, D.; Charleux, B. Nitroxide-mediated polymerization. Progr. Polym. Sci. 2013, 38, 63-235.

18. Moad, G.; Rizzardo, E.; Solomon, D.H. A product study of the nitroxide inhibited thermal polymerization of styrene. Polym. Bull. 1982, 6, 589-593.

19. Georges, M.K.; Veregin, R.P.N.; Kazmaier, P.M.; Hamer, G.K. Narrow molecular-weight resins by a free-radical polymerization process. Macromolecules 1993, 26, 2987-2988.

20. Hawker, C.J. Molecular weight control by a "living" free-radical polymerization process. J. Am. Chem. Soc. 1994, 116, 11185-11186.

21. Hawker, C.J.; Barclay, G.G.; Orellana, A.; Dao, J.; Devonport, W. Initiating systems for nitroxide-mediated "living" free radical polymerizations: Synthesis and evaluation. Macromolecules 1996, 29, 5245-5254.

22. Fischer, H. The persistent radical effect in "living" radical polymerization. Macromolecules 1997, 30, 5666-5672.

23. Fischer, H. The persistent radical effect in controlled radical polymerizations. J. Polym. Sci. Polym. Chem. 1999, 37, 1885-1901.

24. Tang, W.; Tsarevsky, N.V.; Matyjaszewski, K. Determination of equilibrium constants for atom transfer radical polymerization. J. Am. Chem. Soc. 2006, 128, 1598-1604.

25. Toloza Porras, C.; D'hooge, D.R.; van Steenberge, P.H.M.; Reyniers, M.-F.; Marin, G.B. A theoretical exploration of the potential of icar atrp for one- and two-pot synthesis of well-defined diblock copolymers. Macromol. React. Eng. 2013, 7, 311-326.

26. Jakubowski, W.; Matyjaszewski, K. Activator generated by electron transfer for atom transfer radical polymerization. Macromolecules 2005, 38, 4139-4146.

27. Jakubowski, W.; Min, K.; Matyjaszewski, K. Activators regenerated by electron transfer for atom transfer radical polymerization of styrene. Macromolecules 2005, 39, 39-45.

28. D'hooge, D.R.; Konkolewicz, D.; Reyniers, M.-F.; Marin, G.B.; Matyjaszewski, K. Kinetic modeling of ICAR ATRP. Macromol. Theory Simul. 2012, 21, 52-69. 
29. Mohammad Rabea, A.; Zhu, S. Achieving high-conversion bulk ATRP with good livingness and well controlled by design and optimization of polymerization temperature profile. Macromol. React. Eng. 2014, 8, 771-776.

30. Van Steenberge, P.H.M.; D’hooge, D.R.; Wang, Y.; Zhong, M.J.; Reyniers, M.F.; Konkolewicz, D.; Matyjaszewski, K.; Marin, G.B. Linear gradient quality of ATRP copolymers. Macromolecules 2012, 45, 8519-8531.

31. Toloza Porras, C.; D’hooge, D.R.; Reyniers, M.F.; Marin, G.B. Computer-aided optimization of conditions for fast and controlled ICAR ATRP of $N$-butyl acrylate. Macromol. Theory Simul. 2013, 22, 136-149.

32. D'hooge, D.; Van Steenberge, P.; Reyniers, M.-F.; Marin, G. Fed-batch control and visualization of monomer sequences of individual ICAR ATRP gradient copolymer chains. Polymers 2014, 6 , 1074-1095.

33. Chan, N.; Cunningham, M.F.; Hutchinson, R.A. Copper mediated controlled radical polymerization of methyl acrylate in the presence of ascorbic acid in a continuous tubular reactor. Polym. Chem. 2012, 3, 1322-1333.

34. Chan, N.; Meuldijk, J.; Cunningham, M.F.; Hutchinson, R.A. Continuous ARGET ATRP of methyl methacrylate and butyl acrylate in a stirred tank reactor. Ind. Eng. Chem. Res. 2013, 52, 11931-11942.

35. Greszta, D.; Matyjaszewski, K. Mechanism of controlled/“living” radical polymerization of styrene in the presence of nitroxyl radicals. Kinetics and simulations. Macromolecules 1996, 29, 7661-7670.

36. Solomon, D.H.; Rizzardo, E.; Cacioli, P. Polymerization Process and Polymers Produced Thereby. U.S. Patent 4,581,429, 8 April 1986.

37. Bertin, D.; Gigmes, D.; Marque, S.R.A.; Tordo, P. Kinetic subtleties of nitroxide mediated polymerization. Chem. Soc. Rev. 2011, 40, 2189-2198.

38. Fukuda, T.; Goto, A.; Ohno, K. Mechanisms and kinetics of living radical polymerizations. Macromol. Rapid Commun. 2000, 21, 151-165.

39. Gigmes, D.; Bertin, D.; Lefay, C.; Guillaneuf, Y. Kinetic modeling of nitroxide-mediated polymerization: Conditions for living and controlled polymerization. Macromol. Theory Simul. 2009, 18, 402-419.

40. Lutz, J.F.; Lacroix-Desmazes, P.; Boutevin, B. The persistent radical effect in nitroxide mediated polymerization: Experimental validity. Macromol. Rapid Commun. 2001, 22, 189-193.

41. Hernandez-Ortiz, J.C.; Vivaldo-Lima, E.; Lona, L.M.F.; McManus, N.T.; Penlidis, A. Modeling of the nitroxide-mediated radical copolymerization of styrene and divinylbenzene. Macromol. React. Eng. 2009, 3, 288-311.

42. Saldívar-Guerra, E.; Bonilla, J.; Becerril, F.; Zacahua, G.; Albores-Velasco, M.; Alexander-Katz, R.; Flores-Santos, L.; Alexandrova, L. On the nitroxide quasi-equilibrium in the alkoxyamine-mediated radical polymerization of styrene. Macromol. Theory Simul. 2006, 15, 163-175.

43. Tang, W.; Fukuda, T.; Matyjaszewski, K. Reevaluation of persistent radical effect in NMP. Macromolecules 2006, 39, 4332-4337.

44. Benoit, D.; Chaplinski, V.; Braslau, R.; Hawker, C.J. Development of a universal alkoxyamine for "living" free radical polymerizations. J. Am. Chem. Soc. 1999, 121, 3904-3920. 
45. Grimaldi, S.; Finet, J.-P.; le Moigne, F.; Zeghdaoui, A.; Tordo, P.; Benoit, D.; Fontanille, M.; Gnanou, Y. Acyclic $\beta$-phosphonylated nitroxides: A new series of counter-radicals for "living"/controlled free radical polymerization. Macromolecules 2000, 33, 1141-1147.

46. Greszta, D.; Matyjaszewski, K. Tempo-mediated polymerization of styrene: Rate enhancement with dicumyl peroxide. J. Polym. Sci. A Polym. Chem. 1997, 35, 1857-1861.

47. He, J.P.; Chen, J.M.; Li, L.; Pan, J.Y.; Li, C.M.; Cao, J.Z.; Tao, Y.F.; Hua, F.J.; Yang, Y.L.; McKee, G.E.; et al. Rate enhancement of nitroxide-mediated living free-radical polymerization by continuous addition of initiator. Polymer 2000, 41, 4573-4577.

48. Malmström, E.; Miller, R.D.; Hawker, C.J. Development of a new class of rate-accelerating additives for nitroxide-mediated "living" free radical polymerization. Tetrahedron 1997, 53, $15225-15236$.

49. Grubbs, R.B. Nitroxide-mediated radical polymerization: Limitations and versatility. Polym. Rev. 2011, 51, 104-137.

50. Nayak, A.; Gupta, S.K. Multi-objective optimization of semi-batch copolymerization reactors using adaptations of genetic algorithm. Macromol. Theory Simul. 2004, 13, 73-85.

51. Deb, K.; Kalyanmoy, D. Multi-Objective Optimization Using Evolutionary Algorithms; John Wiley \& Sons: Hoboken, NJ, USA, 2001; p. 518.

52. Coello Coello, C.A. Evolutionary multi-objective optimization: A historical view of the field. Comput. Intell. Mag. IEEE 2006, 1, 28-36.

53. Srinivas, N.; Deb, K. Muiltiobjective optimization using nondominated sorting in genetic algorithms. Evolut. Comput. 1994, 2, 221-248.

54. Deb, K.; Pratap, A.; Agarwal, S.; Meyarivan, T. A fast and elitist multiobjective genetic algorithm: NSGA-II. IEEE Trans. Evol. Comput. 2002, 6, 182-197.

55. Mitra, K.; Deb, K.; Gupta, S.K. Multiobjective dynamic optimization of an industrial nylon 6 semibatch reactor using genetic algorithm. J. Appl. Polym. Sci. 1998, 69, 69-87.

56. Bhaskar, V.; Gupta, S.K.; Ray, A.K. Multiobjective optimization of an industrial wiped film poly(ethylene terephthalate) reactor: Some further insights. Comput. Chem. Eng. 2001, 25, 391-407.

57. Mitra, K.; Majumdar, S.; Raha, S. Multiobjective dynamic optimization of a semi-batch epoxy polymerization process. Comput. Chem. Eng. 2004, 28, 2583-2594.

58. Garg, S.; Gupta, S.K. Multiobjective optimization of a free radical bulk polymerization reactor using genetic algorithm. Macromol. Theory Simul. 1999, 8, 46-53.

59. Benyahia, B.; Latifi, M.A.; Fonteix, C.; Pla, F. Multicriteria dynamic optimization of an emulsion copolymerization reactor. Comput. Chem. Eng. 2011, 35, 2886-2895.

60. Massebeuf, S.; Fonteix, C.; Hoppe, S.; Pla, F. Development of new concepts for the control of polymerization processes: Multiobjective optimization and decision engineering. I. Application to emulsion homopolymerization of styrene. J. Appl. Polym. Sci. 2003, 87, 2383-2396.

61. Fierens, S.K.; D’hooge, D.R.; van Steenberge, P.H.M.; Reyniers, M.-F.; Marin, G.B. MAMA-SG1 initiated nitroxide mediated polymerization of styrene: From arrhenius parameters to model-based design. Chem. Eng. J. 2014, doi:10.1016/j.cej.2014.09.024. 
62. Bentein, L.; D’hooge, D.R.; Reyniers, M.F.; Marin, G.B. Kinetic modeling as a tool to understand and improve the nitroxide mediated polymerization of styrene. Macromol. Theory Simul. 2011, 20, 238-265.

63. Petzold, L. Automatic selection of methods for solving stiff and nonstiff systems of ordinary differential-equations. SIAM J. Sci. Statist. Comput. 1983, 4, 136-148.

64. Payne, K.A.; D’hooge, D.R.; van Steenberge, P.H.M.; Reyniers, M.F.; Cunningham, M.F.; Hutchinson, R.A.; Marin, G.B. Arget atrp of butyl methacrylate: Utilizing kinetic modeling to understand experimental trends. Macromolecules 2013, 46, 3828-3840.

65. Derboven, P.; D’hooge, D.R.; Reyniers, M.-F.; Marin, G.B.; Barner-Kowollik, C. The long and the short of radical polymerization. Macromolecules 2015, 48, 492-501.

66. Tang, W.; Matyjaszewski, K. Kinetic modeling of normal ATRP, normal ATRP with [CuII] 0, reverse ATRP and SR\&NI ATRP. Macromol. Theory Simul. 2008, 17, 359-375.

67. Mayo, F.R. Chain transfer in the polymerization of styrene. VIII. Chain transfer with bromobenzene and mechanism of thermal initiation1. J. Am. Chem. Soc. 1953, 75, 6133-6141.

68. Zetterlund, P.B.; Saka, Y.; McHale, R.; Nakamura, T.; Aldabbagh, F.; Okubo, M. Nitroxide-mediated radical polymerization of styrene: Experimental evidence of chain transfer to monomer. Polymer 2006, 47, 7900-7908.

69. Johnston-Hall, G.; Monteiro, M.J. Bimolecular radical termination: New perspectives and insights. J. Polym. Sci. Polym. Chem. 2008, 46, 3155-3173.

70. Johnston-Hall, G.; Monteiro, M.J. Kinetic simulations of atom transfer radical polymerization (ATRP) in light of chain length dependent termination. Macromol. Theory Simul. 2010, 19, 387-393.

71. Matyjaszewski, K. Mechanistic and synthetic aspects of atom transfer radical polymerization. J. Macromol. Sci. A 1997, 34, 1785-1801.

72. Goto, A.; Fukuda, T. Kinetics of living radical polymerization. Progr. Polym. Sci. 2004, 29, 329-385.

73. Zhong, M.J.; Matyjaszewski, K. How fast can a CRP be conducted with preserved chain end functionality? Macromolecules 2011, 44, 2668-2677.

74. Woloszyn, J.D.; McAuley, K.B. Application of parameter selection and estimation techniques in a thermal styrene polymerization model. Macromol. React. Eng. 2011, 5, 453-466.

75. Belincanta-Ximenes, J.; Mesa, P.V.R.; Lona, L.M.F.; Vivaldo-Lima, E.; McManus, N.T.; Penlidis, A. Simulation of styrene polymerization by monomolecular and bimolecular nitroxide-mediated radical processes over a range of reaction conditions. Macromol. Theory Simul. 2007, 16, 194-208.

(C) 2015 by the authors; licensee MDPI, Basel, Switzerland. This article is an open access article distributed under the terms and conditions of the Creative Commons Attribution license (http://creativecommons.org/licenses/by/4.0/). 